\title{
Potential of Ultraviolet Wide-Field Imaging and Multiphoton Microscopy for Analysis of Dehydroergosterol in Cellular Membranes
}

\author{
DANIEL WÜSTNER, ${ }^{1 *}$ JONATHAN R. BREWER, ${ }^{1,2}$ LUIS BAGATOLLI, ${ }^{1,2}$ AND DANIEL SAGE ${ }^{3}$ \\ ${ }^{1}$ Department of Biochemistry and Molecular Biology, University of Southern Denmark, DK-5230 Odense M, Denmark \\ ${ }^{2}$ Membrane Biophysics and Biophotonics Group, MEMPHYS Center for Biomembrane Physics, University of Southern Denmark, \\ DK-5230 Odense M, Denmark \\ ${ }^{3}$ Biomedical Imaging Group, Ecole Polytechnique Fédérale de Lausanne (EPFL), CH-1015 Lausanne, Switzerland
}

\begin{abstract}
KEY WORDS cholesterol; intracellular transport; microdomain; fluorescence; image correlation
ABSTRACT Dehydroergosterol (DHE) is an intrinsically fluorescent sterol with absorption/ emission in the ultraviolet (UV) region and biophysical properties similar to those of cholesterol. We compared the potential of UV-sensitive low-light-level wide-field (UV-WF) imaging with that of multiphoton (MP) excitation microscopy to monitor DHE in living cells. Significantly reduced photobleaching in MP microscopy of DHE enabled us to acquire three-dimensional $z$-stacks of DHEstained cells and to obtain high-resolution maps of DHE in surface ruffles, nanotubes, and the apical membrane of epithelial cells. We found that the lateral resolution of MP microscopy is $\sim 1.5$-fold higher than that of UV-WF deconvolution microscopy, allowing for improved spatiotemporal analysis of plasma membrane sterol distribution. Surface intensity patterns of DHE with a diameter of $0.2 \mu \mathrm{m}$ persisting over several minutes could be resolved by MP time-lapse microscopy. Diffusion coefficients of $0.25-\mu \mathrm{m}$-diameter endocytic vesicles containing DHE were determined by MP spatiotemporal image correlation spectroscopy. The requirement of extremely high laser power for visualization of DHE by MP microscopy made this method less potent for multicolor applications with organelle markers like green fluorescent protein-tagged proteins. The signal-to-noise ratio obtainable by UV-WF imaging could be significantly improved by pixelwise bleach rate fitting and calculation of an amplitude image from the decay model and by frame averaging after pixelwise bleaching correction of the image stacks. We conclude that UV-WF imaging and MP microscopy of DHE provide complementary information regarding membrane distribution and intracellular targeting of sterols. Microsc. Res. Tech. 74:92-108, 2011. @ 2010 Wiley-Liss, Inc.
\end{abstract}

\section{INTRODUCTION}

Direct visualization of sterol transport in living cells has been made possible by using the intrinsically fluorescent sterol dehydroergosterol (DHE) as a close cholesterol analog combined with appropriate microscope instrumentation (Mukherjee et al., 1998). While the physicochemical properties of DHE resemble those of cholesterol very well, its fluorescence yield is low, if a comparison with common fluorescence tags like nitrobenzoxadiazole (NBD) is made. Attaching the NBD fluorophore to cholesterol, however, was found to dramatically alter the biophysical properties of these probes compared to cholesterol including miss targeting in cells (Mukherjee et al., 1998; Scheidt et al., 2003; Wüstner, 2007a).

When imaging DHE, one has to deal with its very low quantum yield ( $Q=0.04$ in ethanol), low extinction coefficient (11.200 $\mathrm{M}^{-1} \mathrm{~cm}^{-1}$ ), rapid photobleaching, and, most importantly, the fact that it excites at 330 $\mathrm{nm}$ and emits at 370-400 $\mathrm{nm}$ in lipid bilayers, a spectral region where commercially available fluorescence microscopes have low light throughput. We have shown that one can load DHE onto methyl- $\beta$-cyclodextrin (MCD) and label cells efficiently and exclusively at the plasma membrane with this DHE/MCD complex. This method allowed for pulse-chase studies of intracellular sterol transport in various cell types employing a widefield (WF) fluorescence microscope with ultraviolet (UV)-optimized optics and sensitive CCD detectors (Hao et al., 2002; Hartwig Petersen et al., 2008; Wüstner, 2005; Wüstner and Færgeman, 2008a,b; Wüstner et al., 2002, 2005). On the other hand, Schroeder and coworkers pioneered in using multiphoton (MP) excitation microscopy for imaging of DHE in living fibroblasts with an excitation wavelength of 900-930 nm (Frolov et al., 2000; McIntosh et al., 2003; Zhang et al., 2005). Since different biological questions have been addressed in most of these studies and different label-

\footnotetext{
Additional Supporting Information may be found in the online version of this article.

*Correspondence to: Daniel Wüstner, Department of Biochemistry and Molecular Biology, University of Southern Denmark, Campusvej 55, DK-5230 Odense M, Denmark. E-mail: wuestner@bmb.sdu.dk

Received 12 October 2009; accepted in revised form 9 April 2010

Contract grant sponsors: Hjerteforeningen (the Danish Heart Association); Diabetesforeningen (the Diabetes Foundation); Forskningsstyrelsen (the Danish Research agency), Forskningsrådet for Natur og Univers (FNU); Forskningsrådet for Sundhed og Sygdom (Denmark); Forskningsrådet for Natur og Univers (Denmark); The Danish National Research Foundation

DOI 10.1002/jemt.20878

Published online 25 May 2010 in Wiley Online Library (wileyonlinelibrary.com).
} 
ing protocols have been used, no direct comparison between both methods could be drawn so far.

Here, we have compared both imaging methods in living Chinese hamster ovarian (CHO) cells, human hepatoma HepG2 cells, and rat hepatoma cells using our pulse-labeling protocol with DHE/MCD. We analyzed intracellular and plasma membrane distribution of DHE, image quality, acquisition speed, and potential for multicolor imaging applications. Our results provide a toolbox for researchers in the field of sterol trafficking and UV microscopy in general. Moreover, we introduce MP spatiotemporal image correlation spectroscopy (STICS) to determine membrane distribution of DHE and mobility of DHE-containing vesicles in living cells. Finally, we assessed the potential of pixelwise bleach rate fitting to improve image quality in UV-sensitive low-light-level WF (UV-WF) microscopy of DHE in living cells.

\section{MATERIALS AND METHODS Reagents}

Fetal calf serum (FCS) and DMEM were obtained from GIBCO BRL (Life Technologies, Paisley, Scotland). All other chemicals were obtained from Sigma Aldrich Chemical (St Louis, MO). Buffer medium contained $150 \mathrm{mM} \mathrm{NaCl}, 5 \mathrm{mM} \mathrm{KCl}, 1 \mathrm{mM} \mathrm{CaCl}, 1 \mathrm{mM}$ $\mathrm{MgCl}_{2}, 5 \mathrm{mM}$ glucose, and $20 \mathrm{mM}$ HEPES ( $\left.\mathrm{pH} 7.4\right)$ as described (Wüstner et al., 2002).

\section{Cell Culture}

HepG2 cells and McArdle RH7777 (McA) cells were grown in DMEM with $4.5 \mathrm{~g} / \mathrm{L}$ glucose, supplemented with $10 \%$ heat-inactivated FCS and antibiotics. Cells were routinely passaged in plastic tissue culture dishes. CHO cells were grown in bicarbonate-buffered Ham's F-12 medium, supplemented with 5\% FCS and antibiotics. For experiments, cells were plated onto glass cover slips coated with poly-D-lysine (Salzmann and Maxfield, 1989). HepG2 cells were used after reaching the highest degree of polarization as described previously (Wüstner et al., 2001).

\section{Labeling of Cells with DHE}

A stock solution of DHE ( $5 \mathrm{mM}$ ), from Sigma Aldrich Chemical, was made in ethanol and stored under nitrogen at $-80^{\circ} \mathrm{C}$. For staining cells, a DHE labeling solution containing DHE/MCD was generated as described (Hao et al., 2002; Wüstner et al., 2002). Cells were pulse labeled for 1-2 min with DHE/MCD, washed with buffer medium, and either imaged directly or chased for another $30 \mathrm{~min}$ at $37^{\circ} \mathrm{C}$ (Wüstner, 2007b; Wüstner and Færgeman, 2008a).

\section{WF Epifluorescence Microscopy and Image Deconvolution}

WF fluorescence microscopy and digital image acquisition were carried out using a Leica DMIRBE microscope with a $63 \times, 1.4$ numerical aperture (NA) oil immersion objective (Leica Lasertechnik, Wetzlar, Germany), equipped with a Orca BT512 4-stage peltier and water-cooled $\left(-80^{\circ} \mathrm{C}\right) \mathrm{CCD}$ camera (Hamamatsu Photonics, Hamamatsu City, Japan) and a Lambda SC smartshutter (Sutter Instrument, Novato, CA), driven by ImagePro Plus and ScopePro (Media Cybernetics, Silver Spring, MD). Optical components and excitation source were described previously (Wüstner, 2007b). $\mathrm{DHE}$ in its monomeric form was imaged in the UV using a specially designed filter cube obtained from Chroma Technology (Brattleboro, VT) with 335-nm (20$\mathrm{nm}$ bandpass) excitation filter, 365-nm dichroic mirror, and 405-nm (40-nm bandpass) emission filter. To improve resolution and image quality, we applied either two-dimensional (2D) deconvolution/deblurring on single planes or maximum likelihood 3D deconvolution using the Huygens software (Scientific Volume Imaging, Hilversum, The Netherlands) as described (Wüstner, 2007b; Wüstner and Færgeman, 2008a,b).

\section{MP Fluorescence Microscopy of DHE}

MP excitation fluorescence microscopy measurements of DHE were done using a custom-built microscope. This setup was constructed using an Olympus IX70 microscope. The objective used was a $60 \times$ water immersion objective with a NA of 1.2. The excitation light source was a femtosecond Ti:Sa laser (Broadband Mai Tai XF W25, with a $10 \mathrm{~W}$ Millennia pump laser, 80 $\mathrm{MHz}$ pulse frequency, tunable excitation range 710 980 nm; Spectra Physics, Mountain View, CA), and the excitation wavelength used was $930 \mathrm{~nm}$ and was circularly polarized. For DHE measurements, the fluorescence signal was collected using a bandpass filter of $377 \pm 25 \mathrm{~nm}$ (BrightLine HC). The light was detected by a photomultiplier tube (Hamamatsu H7422P-40) operated in photon counting mode. The data were acquired using simFCS software developed by the Laboratory for Fluorescence Dynamics University of California, Irvine.

\section{Assessment of Image Quality by Frame Averaging in Fluorescence Microscopy of DHE}

To assess the improvement of image quality by frame averaging, we calculated an estimate for the root mean square (RMS) noise per image in the following manner: first, a $3 \times 3$ median filter was applied to the frameaveraged image to remove subresolution fluctuations due to noise, and this filtered image was subtracted from the original frame-averaged image. The obtained difference image was squared, and the integrated intensity calculated and divided by the pixel number in the image to obtain the mean intensity (Murray, 1998). The square root of this mean difference in intensity between raw and median-filtered image gave the RMS noise, which was plotted as function of the number of averaged frames (see Fig. 2, Results section). Image fidelity can also be assessed by calculating the signal-tonoise ratio (SNR). The SNR can be approximated by dividing the estimated RMS noise image into the original image (Murray, 1998). To compare a reference image $r(x, y)$ with a noisy test image $t(x, y)$ one often uses the peak-SNR (PSNR) as a measure of restoration quality (Gonzalez and Woods, 2002). The PSNR was calculated between the last frame-averaged image as reference image and successive frame averages as test image in both imaging modalities and between fitted amplitude (reference) and raw or bleach-corrected stack (test) in case of UV-WF imaging (see the following text). For these calculations, we used a plugin to 
ImageJ, developed by one of us (DS). This plugin is freely available at http://bigwww.epfl.ch/sage/soft/snr/.

PSNR $=10 \cdot \log _{10}\left[\frac{\max (r(x, y))^{2}}{\frac{1}{M \cdot N} \sum_{x=0}^{M-1} \sum_{y=0}^{N-1}(r(x, y)-t(x, y))^{2}}\right]$

\section{Sterol Distribution and Dynamics Analyzed by MP Excitation Microscopy Combined With Image Correlation Spectroscopy}

Spatiotemporal sterol intensity patterns were calculated from MP time-lapse sequences of DHE-stained cells in several ways:

1. For assessment of vesicle dynamics, STICS was applied to time-lapse sequences of DHE-stained HepG2 cells according to Kolin and Wiseman (2007), but with tools in ImageJ (developed at the U.S. National Institutes of Health and available on the Internet at http://rsb.info.nih.gov/ij). The spatiotemporal image correlation function of a fluorescence intensity fluctuation $\delta F(x, y, t)=F(x, y, t)-\langle F(t)\rangle$ is given by (Brown et al., 2006; Hebert et al., 2005a; Kolin and Wiseman, 2007)

$$
r(\xi, \eta, \tau)=\frac{\langle\delta F(x, y, t) \cdot \delta F(x+\xi, y+\eta, t+\tau)\rangle}{\langle F(t)\rangle^{2}}
$$

It was calculated for a $64 \times 64$ pixel region using the fast Fourier transform implemented in ImageJ. In detail, a stack of a recorded time-lapse sequence of DHE-stained cells (stack 1) was copied (stack 2) and the two stacks were correlated framewise in ImageJ using the fast Fourier transform implemented in ImageJ, getting a stack of all spatial correlations with no time lag $(\tau=0)$. The average of this stack was calculated and stored. Next, the procedure was repeated for a time lag $1(\tau=1)$ by spatially correlating plane $i$ for $i$ $=1, \ldots, N ; N$, number of frames in stack 1 with plane $(i+1)$ in stack 2 ; the average of the resulting correlation stack was stored and the procedure repeated for $i$ $=2$, and so on. At the end, all average images obtained from the measured correlation stacks were recombined in a single stack and divided by the squared mean intensity of the whole time-lapse sequence. In this way, we obtained the spatiotemporal correlation of the timelapse sequence. Alternatively, the spatiotemporal image correlation function was calculated with plugins to ImageJ developed and kindly provided by Jay Unruh (Stowers Microscopy Center, Stowers Institute, Kansas City, MO). Both methods gave the same results. A line scan along the peak intensity provided the 1D profile of the obtained correlation function, which was fitted to

$$
r(0,0, \tau)=r_{\infty}+\frac{1}{1+\tau \cdot 4 D / \omega_{\mathrm{mp}}^{2}}
$$

after normalizing the temporal image correlation function $r(0,0, \tau)$ to the initial value $r(0,0,0)$ (Kolin and Wiseman, 2007). It has been shown that estimation of the amplitude of the correlation function, but not determination of the diffusion coefficient according to Eq. (3), is very sensitive to photobleaching and SNR (Kolin et al., 2006). Since, imaging of DHE suffers under both problems, we decided to perform this normalization and avoid fitting the amplitude value in this application.

2. For analysis of plasma membrane staining patterns of DHE as measured by MP microscopy, intensity line scans were extracted from the cell perimeter as described (Wüstner and Færgeman, 2008b). From these intensity profiles, spatial and temporal correlations were calculated separately. The spatial autocorrelation function $r(\xi, 0)$ with zero time lag is given by a $1 \mathrm{D}$ version of Eq. (2) (no $y$ or $\eta$ variable) and with $\tau=0$ (Hebert et al., 2005b; Kolin and Wiseman, 2007):

$$
r(\xi, 0)=\frac{\langle\delta F(x, t) \cdot \delta F(x+\xi, t)\rangle}{\langle F(t)\rangle_{t}^{2}}
$$

Temporal correlation of the time-dependent fluorescence intensity profiles of DHE-stained plasma membrane was calculated by a bivariate correlation analysis implemented in SigmaPlot 9.0 (SPSS, Chicago, IL) defining the correlation coefficient $R$ as a function of time, $t$, relative to the first intensity pattern at $t=t_{0}$, as described (Wüstner and Færgeman, 2008b).

$$
R(t)=\frac{\sum\left(\left(x_{i}(t)-\bar{x}(t)\right) \cdot\left(y_{i}(t)-\bar{y}(t)\right)\right)}{\sqrt{\sum\left(x_{i}(t)-\bar{x}(t)\right)^{2} \cdot \sum\left(y_{i}(t)-\bar{y}(t)\right)^{2}}}
$$

where $x_{i}$ and $y_{i}$ are pixel intensities and $\bar{x}$ and $\bar{y}$ are mean intensities measured from outlined plasma membrane segments.

A number and brightness (N\&B) analysis was performed in ImageJ to assess the average or apparent brightness, $B$, of sterol-containing structures at the cell surface (Digman et al., 2008). It is given by

$$
B=\frac{\sigma^{2}}{\langle k\rangle}=\frac{\sum_{i}\left(k_{i}-\langle k\rangle\right)^{2}}{\sum_{i} k_{i}}=\varepsilon+1
$$

Here, $k_{i}$ is the number of photon counts at a particular pixel position, whereas $\langle k\rangle$ and $\sigma^{2}$ represent the first and second moments of the intensity distribution (i.e., the average intensity and variance), respectively. The apparent brightness is, thus, a measure of the intensity variance per pixel normalized to the average intensity. It is related to the molecular brightness, $\varepsilon$, of the particles and is independent of the number of particles. The apparent number of particles, $N$, is calculated from the same parameters according to.

$$
N=\frac{\langle k\rangle^{2}}{\sigma^{2}}=\frac{\varepsilon \cdot n}{\varepsilon+1}
$$

One can see that $N$ is directly proportional to the number of fluorescent particles in a given pixel location.

\section{Pixelwise Bleach-Rate Fitting and Bleaching Correction of UV-WF Stacks}

DHE-stained cells were repeatedly illuminated on a UV-WF microscope, and images were acquired without 
interval to monitor the bleaching of DHE as described (Wüstner et al., 2010). The intensity decay of DHE was fitted on a pixel-by-pixel basis to a monoexponential decay function using our ImageJ plugin PixBleach available at http://bigwww.epfl.ch/algorithms/pixbleach (Wüstner et al., 2010). Here, we extended this plugin to correct for the bleaching process in an additive manner according to

$$
f^{\prime}(x, y, t)=f(x, y, t)+A(x, y) \cdot(1-\exp (-t / \tau(x, y)))
$$

where $f(x, y, t)$ is the input bleach stack as function of pixel coordinates $(x, y)$ and illumination time $t, f^{\prime}(x, y, t)$ is the bleach-corrected stack, $A(x, y)$ is the amplitude and $\tau(x, y)$ is the time constant, as obtained from the pixelwise fit.

\section{RESULTS AND DISCUSSION}

This section is organized as follows. First, we compare the potential of MP microscopy for imaging of DHE with our previously developed UV-WF method. Second, we explain in detail the acquisition process in the MP microscope including frame averaging and provide examples of high-resolution images of DHElabeled cells obtained by this technique. Third, we use time-lapse MP microscopy of DHE to reveal intracellular and surface dynamics of the sterol analyzed by image correlation techniques. Fourth, we compare the potential of both methods for multicolor application and colocalization studies of intracellular DHE. Fifth, we introduce a new method to improve image quality in UV-WF microscopy of DHE despite the high photobleaching of the sterol in this imaging mode. Finally, we compare the impact of frame averaging on image quality in UV-WF and MP microscopy of DHE.

\section{Comparison of UV-WF and MP Excitation Microscopy for Imaging of DHE-Labeled Cells}

CHO cells pulse labeled with DHE/MCD for $1 \mathrm{~min}$ were either directly imaged by UV-WF or MP microscopy (Figs. 1A and 1B) or chased for $30 \mathrm{~min}$ at $37^{\circ} \mathrm{C}$ prior to image acquisition by both methods (Figs. 1C and 1D). Plasma membrane staining and a few endocytic vesicles can be clearly distinguished by both methods at the early time point, whereas after $30 \mathrm{~min}$ incubation prominent vesicle accumulation in the perinuclear region was found. Images acquired with our MP setup (see Materials and Methods) appear sharper, i.e., cell edges are more clearly resolved. This is caused by the intrinsic sectioning effect of the MP microscope greatly reducing the depth of field (DOF) and out-offocus contributions. By comparing DHE-labeled cells with nonlabeled cells, we found that the DHE signal obtained by UV-WF imaging is about five times over autofluorescence of the cells, whereas that obtained by MP excitation microscopy is about three-fold over autofluorescence background (Figs. 1E-1H). While the DHE intensity in UV-WF modus is thus clearly distinguishable from cellular autofluorescence, there are few strongly autofluorescent organelles (probably lysosomes), which can be misinterpreted as weakly fluores-
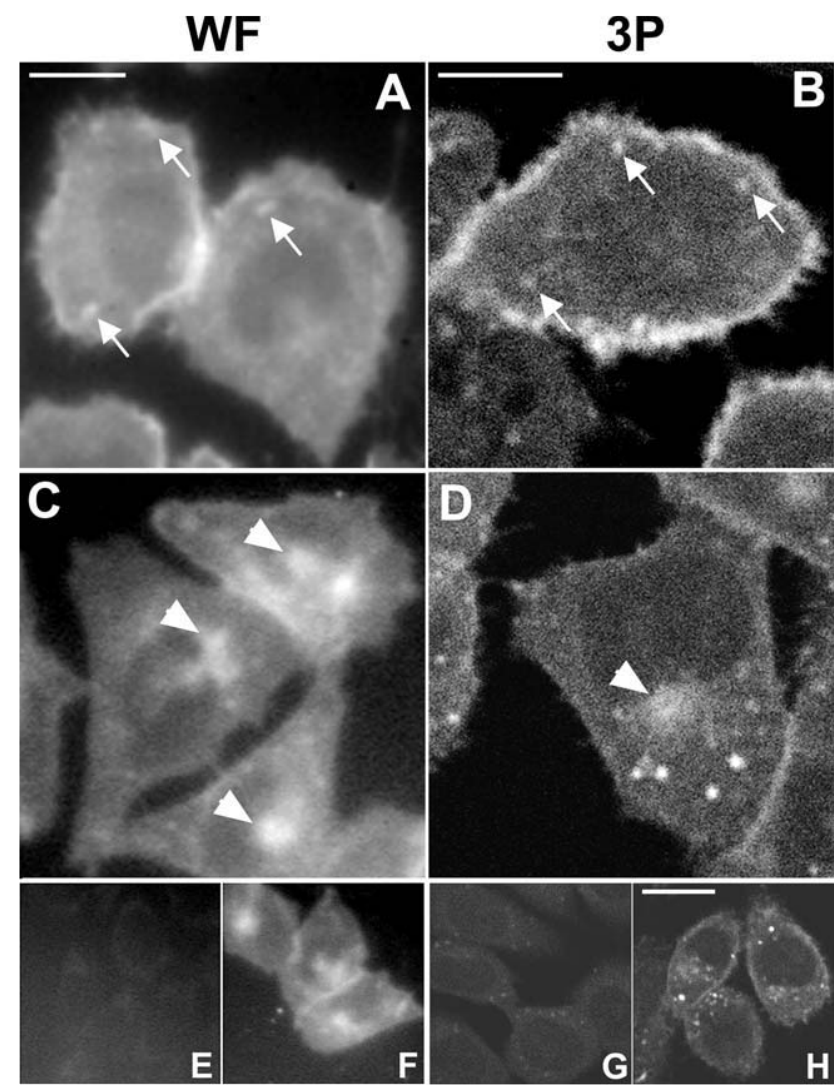

Fig. 1. Distribution of DHE in CHO cells imaged by UV-WF and MP microscopy. A-D: CHO cells were pulse labeled with DHE/MCD for $1 \mathrm{~min}$ at $37^{\circ} \mathrm{C}$, washed, and either imaged directly (A, B) or chased for $30 \mathrm{~min}$ at $37^{\circ} \mathrm{C}$ before imaging $(\mathrm{C}, \mathrm{D})$. DHE-stained cells were either imaged on a WF microscope with an excitation wavelength of 310-330 nm (A, C) or on a MP microscope with an excitation wavelength of $930 \mathrm{~nm}$. In case of UV-WF imaging, a single acquisition is shown, whereas the images obtained by MP microscopy represent averages of 70 (B) or 55 acquisitions (D). In both imaging modes, the plasma membrane, endocytic vesicles (arrows), and the perinuclear ERC (arrowheads) were found to contain detectable amounts of DHE. E-H: Nonlabeled (E, G) or DHE-labeled CHO cells $(\mathrm{F}, \mathrm{H})$ were imaged identically by WF (E, F) or MP microscopy $(\mathrm{G}, \mathrm{H})$. Identical scaling was used for labeled and nonlabeled cells to reveal intensity of autofluorescent structures compared to DHE-containing organelles (see text for further details). Bar, $10 \mu \mathrm{m}$.

cent DHE vesicles in the MP microscope. Thus, UV-WF microscopy of DHE is superior in discriminating DHE signal from cellular autofluorescence in the UV region of the spectrum.

In our setup, acquisition of a high-resolution image of DHE-labeled cells by UV-WF microscopy, as shown in Figures $1 \mathrm{~A}$ and $1 \mathrm{C}$, requires typically $1-2 \mathrm{~s}$ acquisition time. This is in strong contrast to image acquisition for DHE on the MP excitation microscope. A single image obtained by line scanning with $40 \mathrm{~mW}$ power of the Ti-Sapphire laser pulsed with a frequency of 80 $\mathrm{MHz}$ and pixel dwell time of $30 \mu \mathrm{s}$ is of very low contrast (Fig. 2A). Thus, we needed to average many images extending the acquisition time to $100 \mathrm{~s}$ for 50 frames with $2 \mathrm{~s}$ per frame to receive one high-resolution image. The repeated acquisition in the MP microscope was possible by the very low photobleaching of DHE in this imaging modus compared to UV-WF imaging (Fig. 2B). Photobleaching is the photochemical deg- 
A
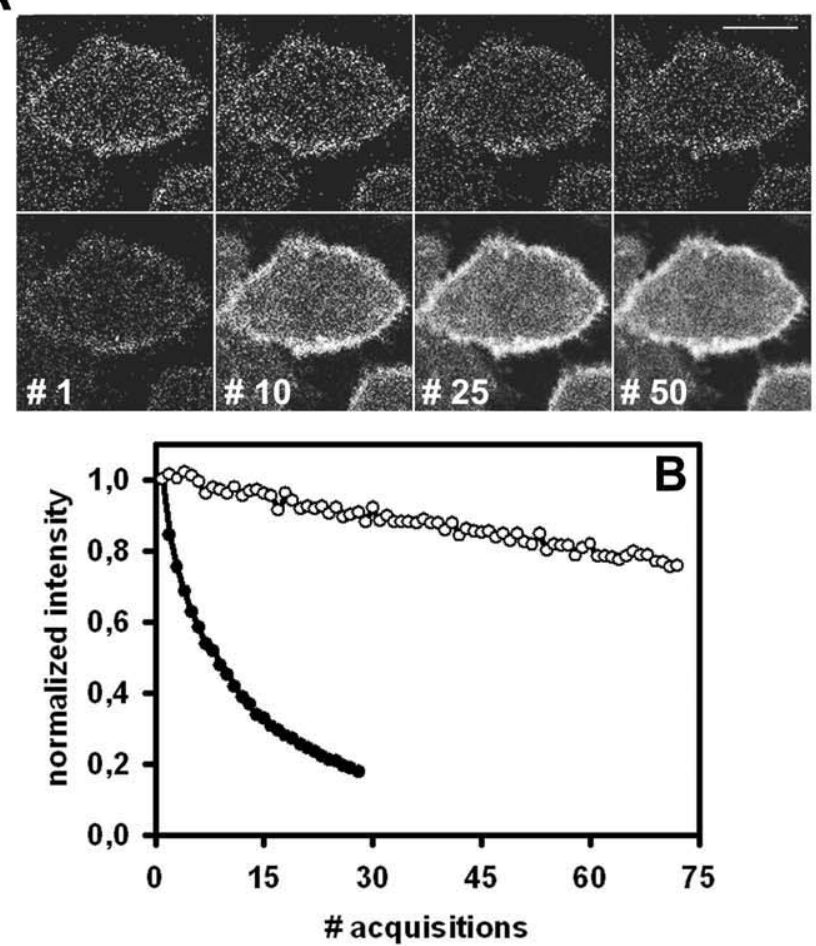

Fig. 2. Frame averaging and photobleaching in MP microscopy of DHE. A: Individual planes (top) and frame averages of 1, 10, 25, and 50 acquisitions (bottom) for the image shown in (B) as obtained by our MP setup. Bar, $10 \mu \mathrm{m}$. B: Quantification of photobleaching of DHE in CHO cells in the MP microscope for a laser power of $60 \mathrm{~mW}$ (open symbols) or the UV-WF microscope and repeated acquisitions with 2-s acquisition time each and a mercury lamp as light source (closed symbols). C: HepG2 cells were pulse labeled with DHE/MCD for $1 \mathrm{~min}$ at

radation of a fluorophore from the excited singlet or triplet state with bleached molecules being removed irreversibly from the excitation/emission photocycle. The reduced photobleaching is a clear advantage of MP microscopy caused by exciting just the DHE molecules in the focus of the infrared laser, where energy density is high enough to have a reasonable probability for threephoton excitation processes to occur (Zipfel et al., 2003). This is in contrast to UV-WF imaging, where a higher DOF and excitation along the whole optical axis results in bleaching of DHE over the entire height of the cells, causing strong bleaching even in regions not being in focus (Fig. 2B, closed circles). The low photodestruction of DHE in MP microscopy allows for averaging over many frames, thereby greatly improving the SNR and thus the resolution (Stelzer, 1998; Wüstner, 2005). Similar attempts in UV-WF imaging of
C
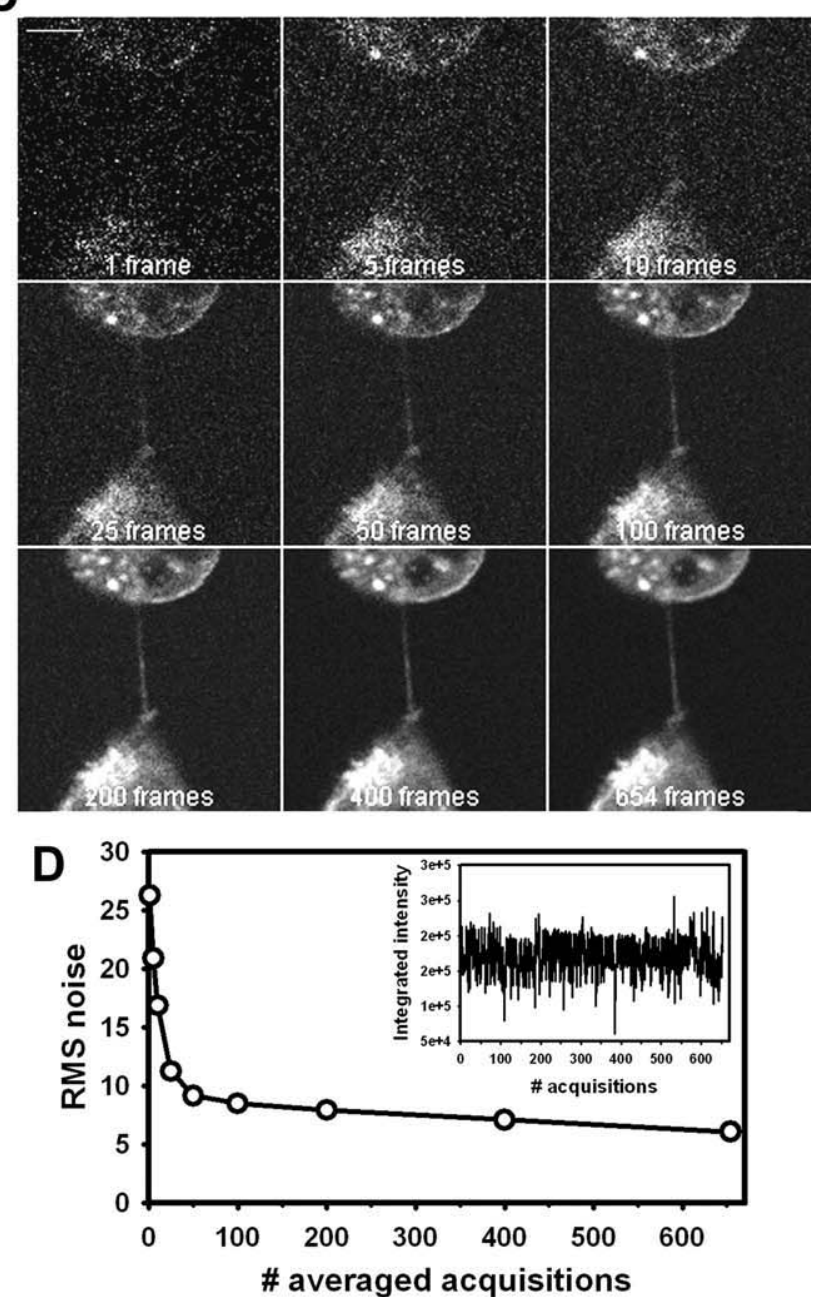

$37^{\circ} \mathrm{C}$, washed, and imaged by MP microscopy using a laser power of $20 \mathrm{~mW}$. A DHE-stained nanotube connecting two cells became visible after averaging 20 planes, but good SNR and image contrast required averaging of at least 100 frames. Bar, $5 \mu \mathrm{m}$. D: RMS noise calculated for the frame-averaged images of (C) as described in Materials and Methods. Inset in D: Intensity in single frames as function of acquisition number was almost constant indicating negligible bleaching due to reduced laser power.

DHE require computational bleaching correction, but are still limited to frame averaging of maximal 20 planes [see following text and Wüstner (2005)]. An extreme example of gain in SNR by frame averaging of MP images is shown in Figures 2C and 2D for a nanotube connecting two HepG2 hepatoma cells. These tiny membranous structures gained large interest recently, because they resemble a new way of cell-to-cell communication (Öfelt et al., 2004; Rustom et al., 2004). Moreover, nanotubes are used by various pathogens to efficiently spread and infect cells in culture (Rustom, 2009). While in a single frame image, visual inspection reveals nothing but noise, after averaging 25 frames a fine intercellular structure becomes apparent, but more than 100 acquisitions are required to resolve the nanotube clearly with high SNR (see also Supporting Information Video). We quantified this process of frame 
averaging by calculating the RMS noise of the images as function of number of averaged frames as explained in Materials and Methods (Fig. 2D). In this particular example, image quality increased tremendously up to 50 averages, and further averaging gave only a minor increase in image quality. For this image, we chose a lower laser power of $20 \mathrm{~mW}$, resulting in almost no bleaching of DHE during the acquisition process (Fig. $2 \mathrm{D}$, inset). One can actually quantify the gain in image quality upon frame averaging by calculating the PSNR as function of the number of averaged frames [see Eq. (1) and the following lines; Fig. 9]. We did this for the two examples of Figures $2 \mathrm{~A}$ and $2 \mathrm{C}$ being acquired at 60 and $20 \mathrm{~mW}$ laser outputs, respectively (see Fig. 9 and below). This analysis revealed that less frame averages are required to obtain a high-quality image in case of larger excitation laser power compared to lower laser power in MP microscopy of DHE. We explain this result by the higher probability of exciting DHE by a MP process when one increases the intensity of the excitation light. This of course goes parallel with a higher bleaching probability (compare open symbols in Fig. 2B with inset in Fig. 2D).

MP excitation microscopy has an intrinsic sectioning capability due to the nonlinear (quadratic for two-photon or cubic for three-photon microscopy) dependence of excitation probability on excitation intensity (Zipfel et al., 2003). By increasing the laser power to $70 \mathrm{~mW}$ and reducing the scan speed to $20 \mu$ s pixel dwell time, we were able to obtain $3 \mathrm{D}$ stacks of cells from single frame acquisitions along the optical axis (Fig. 3A). A $3 \mathrm{D}$ reconstruction reveals the distribution of DHE-containing vesicle populations, including the perinuclear DHE pool resembling mostly the endocytic recycling compartment (ERC) in CHO cells (Fig. 3A, arrows; Hao et al., 2002). We observed DHE in surface protrusions and filopodia along the whole $z$-stack, as exemplified for two planes being acquired $0.5 \mu \mathrm{m}$ apart (Figs. 3B and $\left.3 \mathrm{~B}^{\prime}\right)$. These filopodial structures emanated up to $1.5 \mu \mathrm{m}$ from the cell surface. We observed similar structures in various cell types using our UV-WF microscope setup combined with computational image restoration (Wüstner, 2007b, 2008). Thus, results obtained by both methods suggest that apparently heterogeneous membrane staining of DHE is in fact a consequence of the rough, highly convoluted cell surface. Next, we acquired 50-60 frames of a cell at three selected positions along the optical axis using a lower laser power $(40 \mathrm{~mW})$ and shorter pixel dwell time to reduce photobleaching. Averaging 10 frames at these positions being $2 \mu \mathrm{m}$ apart provided high-resolution images of DHE-labeled CHO cells with several DHE-stained nanotubes connecting cells at different heights (Figs. $3 \mathrm{C}-3 \mathrm{E}$, arrowheads). Thus, we can, for the first time, visualize sterol in intercellular nanotubes at different positions along the optical axis.

HepG2 cells are human liver-derived hepatoma cells, which maintain the ability to polarize and form apical bile canaliculi (BC)-like vacuoles resembling the canaliculi biliferi of the liver (Sormunen et al., 1993). This canalicular network branches through the whole organ and contains the bile fluid secreted by hepatocytes. Polarized HepG2 cells have been shown to be a good model system for studying vectorial hepatic transport, including polarized trafficking of fluorescent
A
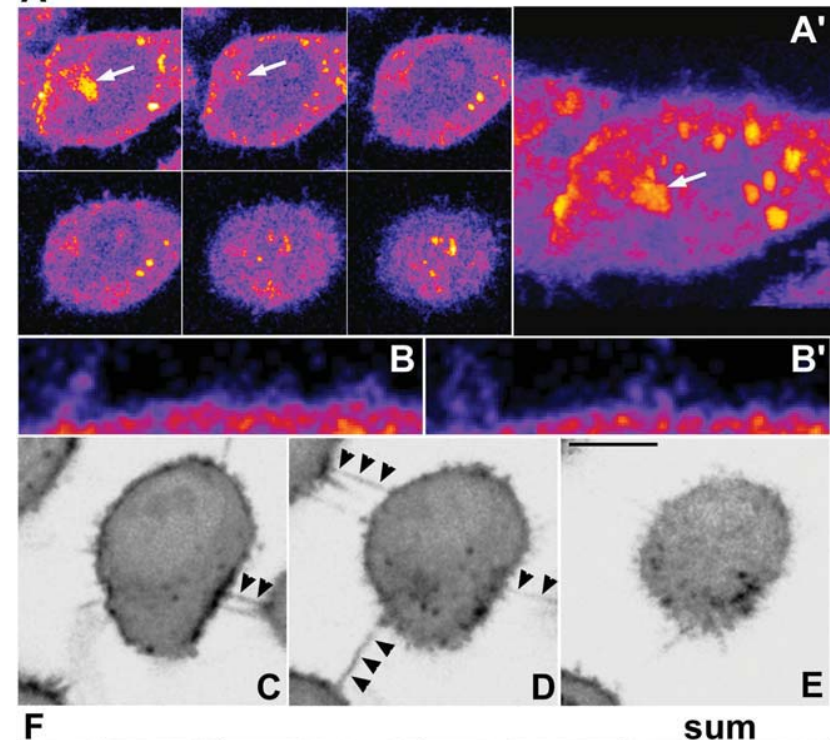

$\mathrm{F}$
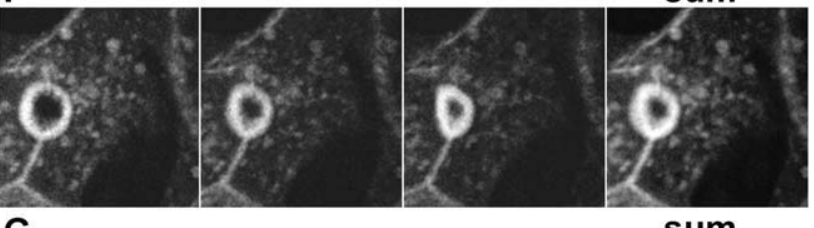

G sum

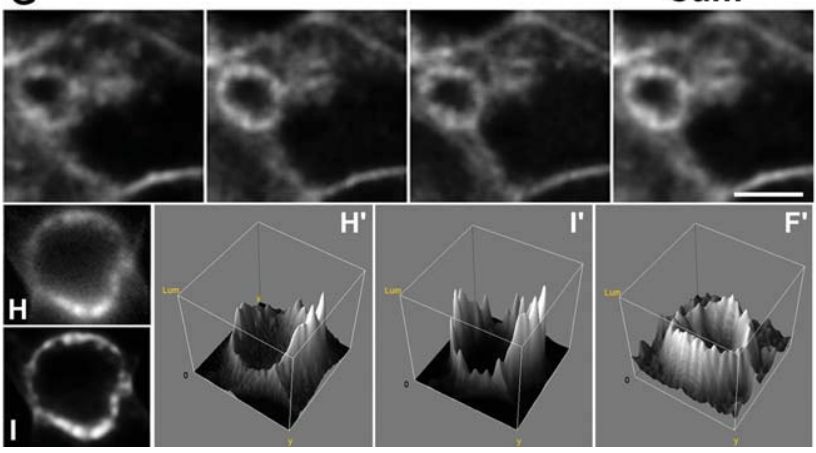

Fig. 3. Three-dimensional imaging of DHE-stained cells by MP and UV-WF microscopy. A-E: CHO cells were pulse labeled with $\mathrm{DHE} / \mathrm{MCD}$ for $1 \mathrm{~min}$ at $37^{\circ} \mathrm{C}$, washed, and chased for $30 \mathrm{~min}$ before imaging on a MP microscope. A, $\mathrm{A}^{\prime}$ : Reconstructed $z$-stack consisting of 36 frames acquired $0.3 \mu \mathrm{m}$ apart. A: Six selected frames; A': 3Dreconstruction in $x z$-view under a 45 degree angle of view [arrows point to the perinuclear ERC pool, previously described in these cells (Hao et al., 2002)]. B, B': Two selected planes acquired $0.6 \mu \mathrm{m}$ apart along the optical $z$-axis show surface protrusions with a length of up to $1.5 \mu \mathrm{m}$. C-E: High-resolution images $2 \mu \mathrm{m}$ apart obtained by averaging 50 frames. Nanotubes connecting cells can be clearly distinguished in (C) and (D) (arrowheads). F-I': HepG2 cells were labeled with DHE as described earlier for CHO cells and imaged on a MP (F) or UV-WF microscope with deconvolution as postprocessing step (G). In both cases, individual planes acquired $0.5 \mu \mathrm{m}$ apart along the $z$-axis are shown; the most right panel shows the sum projection of the other planes. The central ring-like structure resembles the apical biliary canaliculus (BC) formed by connected polarized HepG2 cells. $\mathrm{H}$, I: Another example of a BC imaged on a UV-WF microscope $(\mathrm{H})$ and subsequently deblurred with a $2 \mathrm{D}$ deconvolution routine (I). Three-dimensional profile of the $\mathrm{BC}$ calculated with ImageJ plugins for the $\mathrm{BC}$ shown in $\mathrm{H}\left(\mathrm{H}^{\prime}\right), \mathrm{I}\left(\mathrm{I}^{\prime}\right)$, and $\mathrm{F}\left(\mathrm{F}^{\prime}\right)$. Bar, $10 \mu \mathrm{m}$.

sphingolipids (van IJzendoorn and Hoekstra, 1998, 1999), transmembrane proteins (Slimane et al., 2003; Wojtal et al., 2006) and GPI-anchored proteins (Aït-Sli- 
mane et al., 2009; Slimane et al., 2003) and polarized distribution and sorting of sterols like DHE (Wüstner, 2005, 2007a,b; Wüstner et al., 2002, 2004). Using the MP imaging approach described earlier, we found an enrichment of DHE-containing vesicles in close proximity of the BC in polarized hepatoma HepG2 cells (Fig. 3F). These sterol-rich vesicles belong to a subapical recycling compartment in these cells, as demonstrated previously in colocalization experiments with markers for this compartment using UV-WF microscopy (Wüstner, 2005; Wüstner et al., 2002). For comparison, we labeled HepG2 cells with DHE exactly as described for the MP microscopy experiment and acquired ten planes along the optical axis using our UV-WF microscope followed by bleaching correction and image restoration using a maximum likelihood deconvolution algorithm implemented in Huygens software (Fig. 3G; Wüstner, 2007b; Wüstner and Færgeman, 2008a). Distinction of individual DHE-stained vesicles in the subapical region is much more difficult in images obtained by this method compared to MP microscopy due to lower contrast caused by photobleaching of DHE along the $z$-stack [compare Figs. 3F and $3 \mathrm{G}$, and see additionally Wüstner (2005), Wüstner and Færgeman (2008a), and Wüstner et al. (2002)]. Thus, MP microscopy is better suited to get high-resolution 3D images of DHE-containing endosomes in a crowded cellular environment. We demonstrated previously that folding of the apical canalicular membrane containing DHE and microvilli protruding into the lumen of the BC of HepG2 cells can be resolved by UV-WF microscopy combined with deconvolution (Wüstner, 2005, 2007b). As shown in Figures $3 \mathrm{~F}^{\prime}, 3 \mathrm{H}^{\prime}$, and $3 \mathrm{I}^{\prime}$, both methods allow for revealing the rough topography of the canalicular membrane, causing an apparent lateral enrichment of DHE in subregions of this plasma membrane domain. It has to be noted, however, that in case of MP microscopy, in this particular example, a slight focus drift excluded better imaging of the apical membrane domain. Otherwise, the lateral resolution of the MP was found to be superior compared to UV-WF deconvolution microscopy of DHE (see below).

\section{Visualization and Quantification of Cell Surface Distribution and Dynamics of DHE}

To assess the ability of three-photon microscopy of DHE to resolve sterol surface distribution, we imaged membrane attachment sides of DHE-labeled epithelial HepG2 cells. Ruffles, microridges, and other surface structures labeled with DHE could be clearly resolved in a sum projection of 100 individual frames acquired at the lower surface of an adherent HepG2 cell (Figs. $4 \mathrm{~A}$ and $4 \mathrm{~B}$ ). Beside large, static adhesion patches in the center of the field, small DHE-labeled structures with a diameter below $1.0 \mu \mathrm{m}$ were observed (Figs. 4B4D). This pattern is similar to what we observe in the UV-WF microscope for the same specimen, though the smallest patches appear more blurred in the latter setup, even after applying image deconvolution [see Figs. $2 \mathrm{~B}$ and $6 \mathrm{~F}$ in Wüstner (2007b)]. The smallest patches resolvable by frame-averaged MP excitation microscopy had a size of $240 \mathrm{~nm}$, as inferred from an estimation of the full width at half maximum (FWHM) of a line scan across these structures (Figs. 4C and 4D). Note that the FWHM of $0.1-\mu \mathrm{m}$ fluorescent beads
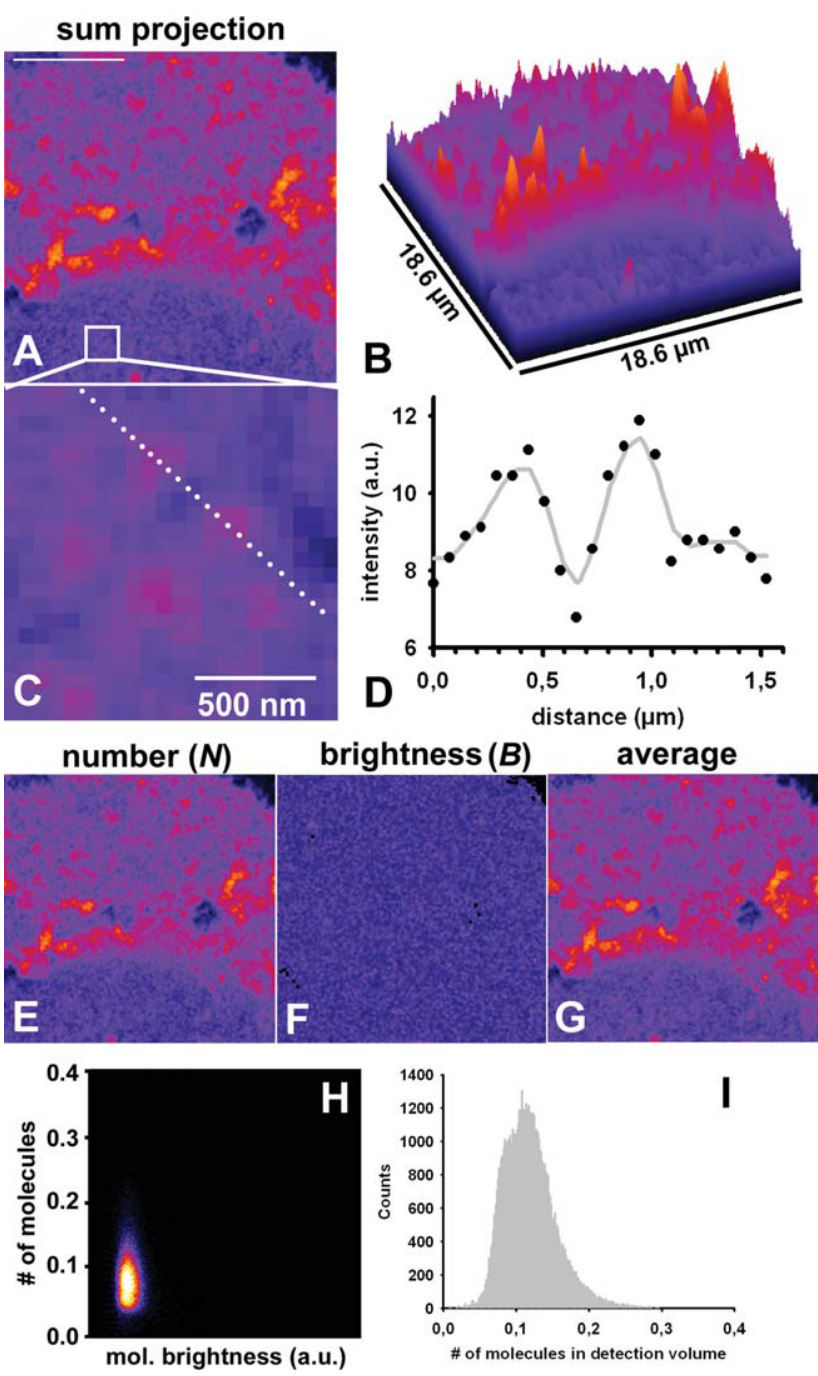

brightness $(B)$

average
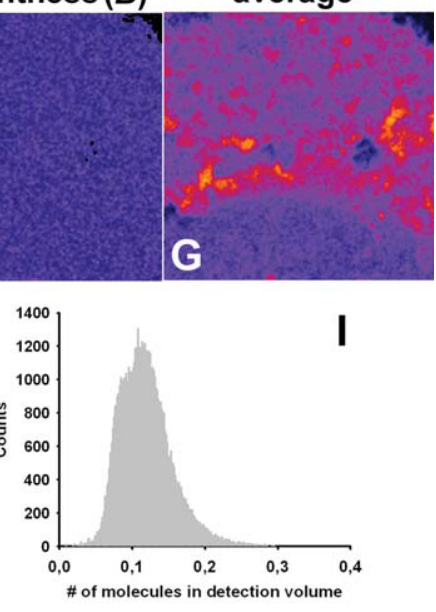

Fig. 4. MP imaging of sterol patterns at cell attachment sites. A HepG2 cells labeled with DHE/MCD for $1 \mathrm{~min}$, washed, and chased for $30 \mathrm{~min}$ at $37^{\circ} \mathrm{C}$. The site of cell attachment to the polylysine substratum was imaged. Sum projection of 100 frames (A) is shown as surface profile in $(\mathbf{B})$. Bar, $3 \mu \mathrm{m}$. C: Zoom of the white box shown in (B) resolves smallest visible structures. Dotted line indicates selected line for calculation of intensity profile over two small patches. D: Intensity profile for the dotted line in (C), black symbols, data; gray line, moving average filter to smooth the data. Half-width of the intensity peaks was 250-300 nm. E-I: Number and brightness analysis according to Eqs. (6) and (7). E: Calculated apparent number; F: Apparent brightness; G: Average intensity; H: Scatter plot of molecular brightness versus average number of molecules; I: Histogram of average number of molecules in the observation volume. See text for further details.

determined in the UV channel of our UV-WF microscope as a measure of lateral resolution of the microscope was $\sim 710 \mathrm{~nm}$ prior to and $390 \mathrm{~nm}$ after deconvolution (Wüstner and Færgeman, 2008a). Thus, the very small DHE-containing patches shown in Figure 4 can probably not be resolved with this precision by UV-WF microscopy, even after applying deconvolution (Wüstner, 2007b). This is confirmed by a similar analysis of the smallest DHE-containing structures along the canalicular membrane (Figs. $3 \mathrm{I}$ and $3 \mathrm{I}^{\prime}$ ) and at cell attachment sites [not shown, but see Fig. 8 in Wüstner 

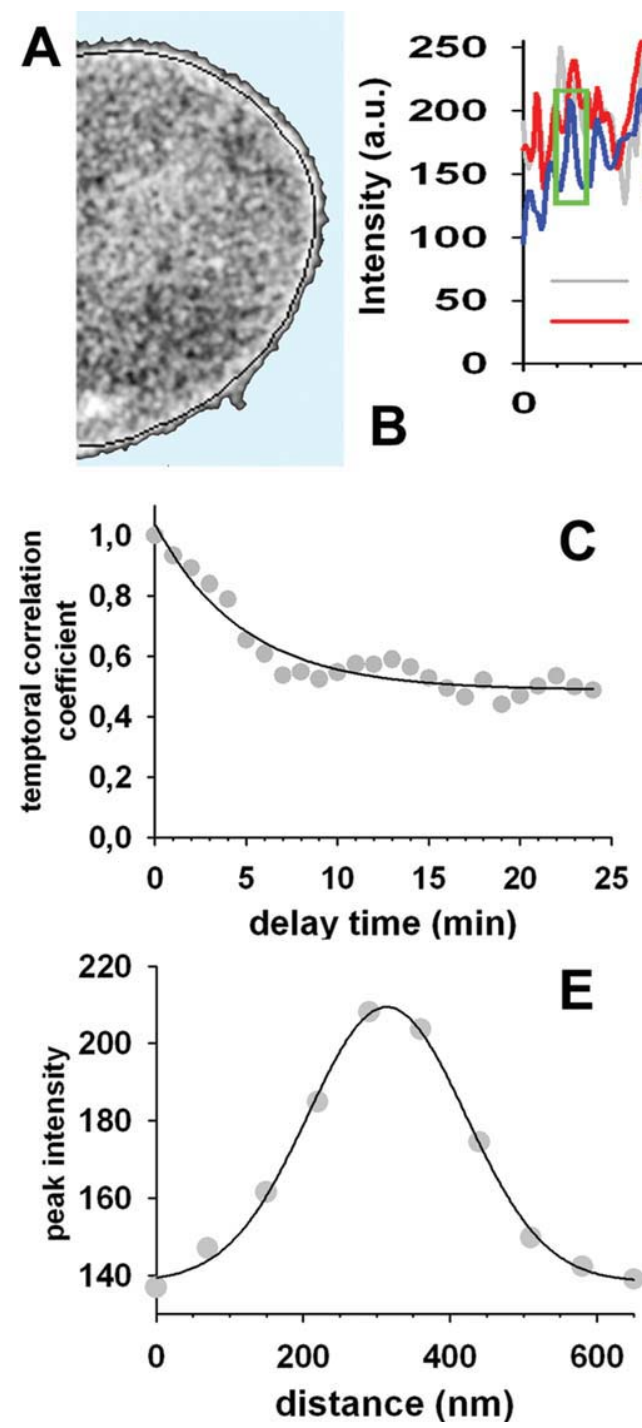

Fig. 5. MP imaging of sterol patterns at the cell periphery. A: HepG2 cells were labeled with DHE/MCD for 1 min, washed, chased for $30 \mathrm{~min}$ at $37^{\circ} \mathrm{C}$, and placed on the temperature-controlled stage of a MP microscope maintained at $35 \pm 1^{\circ} \mathrm{C}$. The perimeter of the cell was imaged over a time course of 25 min with nine averaged frames recorded every $1 \mathrm{~min}$ at $37^{\circ} \mathrm{C}$. A: Representative inverted image of the cell perimeter with drawn line (black) indicating the region for measurement of intensity profiles shown in $(\mathbf{B})$. C: Temporal correlation of the intensity profiles with respect to the line profile at the start of the sequence $(t=0)$, calculated using Eq. (5). D: Spatial autocorrela-

(2007b)] resolvable by UV-WF deconvolution imaging. These patches have a FWHM of 400-450 nm, almost double as large as those recorded by MP microscopy in the same cell type under identical conditions. Thus, MP microscopy significantly improves the lateral resolution and thereby the accuracy of detecting tiny DHEcontaining structures in living cells. Because of the second- and third-order power dependence, the theoretical limit of lateral resolution at an excitation laser wavelength of $\lambda=900 \mathrm{~nm}$ is FWHM $=220$ and $190 \mathrm{~nm}$ for a two- and three-photon instrument, respectively (Schrader et al., 1997). This confirms our experimental findings of improved lateral resolution in MP micros-
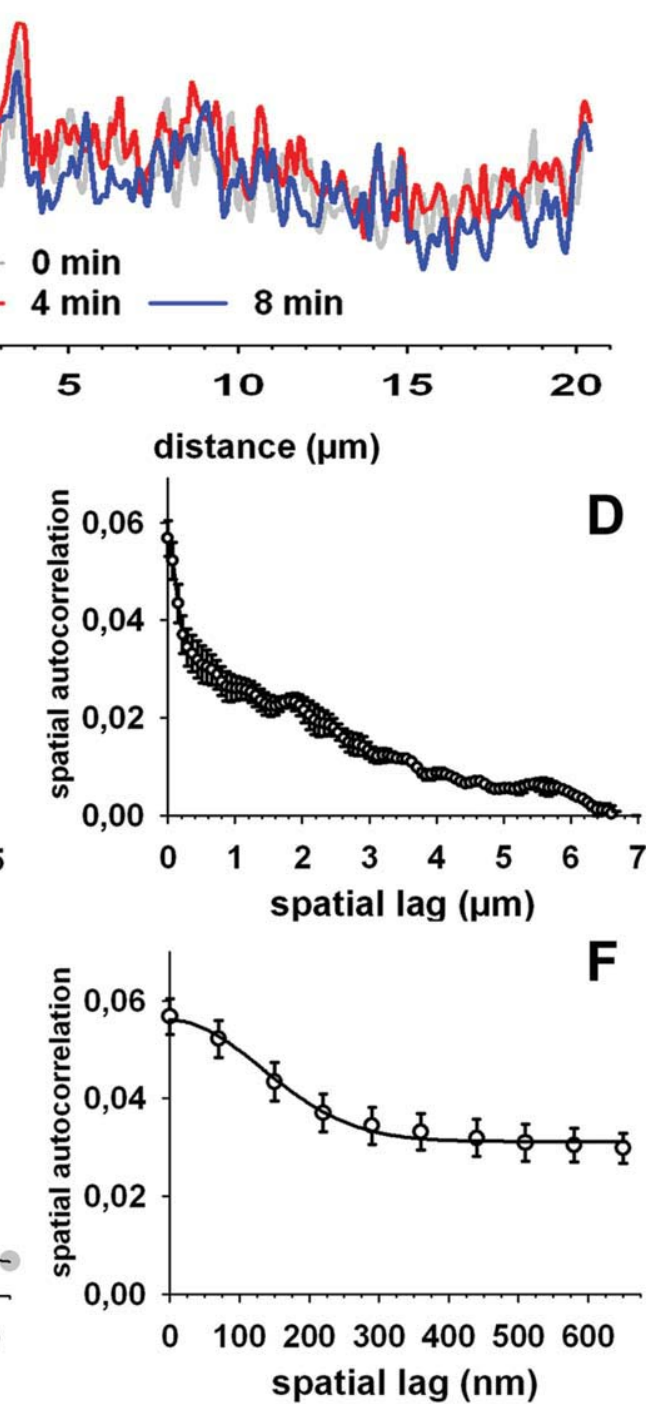

tion calculated for all 25 line profiles and averaged according to Eq. (4) (mean \pm SD). E: Zoom of the green box in (B) for the intensity profile of the 8-min time point (gray symbols) fitted to a Gaussian function of the form $I(x)=I_{0}+a \cdot \exp \left[-0.5 \cdot\left(\frac{x-x_{0}}{b}\right)^{2}\right]$ (black line). From this fit, the FWHM of the peak can be determined to be $235 \mathrm{~nm}$. F : Zoom of the spatial autocorrelation function shown in (D) for the first $0.65 \mu \mathrm{m}$ of spatial lag (open symbols). Black line, fit of Eq. (9) (see Results section) to data. See text for further explanations.

copy of DHE in living cells. To measure the resolution of our system, we recorded images of subresolution quantum dots with an excitation wavelength of $\lambda=$ $930 \mathrm{~nm}$. The particle profile was measured from the acquired images, and a Gaussian function was fitted to it, as indicated in legend to Figure 5. This gave a value of FWHM = $287 \mathrm{~nm}$ (see Supporting Information Fig. S1). The actual lateral resolution in case of sterol imaging might be even better, since quantum dots are likely excited by a two-photon process, and the obtained resolution is accordingly given by the two-photon point spread function (2P-PSF; Schrader et al., 1997). Excitation of DHE at a wavelength of $\lambda=930 \mathrm{~nm}$ is most prob- 
ably a three-photon process, and accordingly a 3P-PSF defines the resolution of the imaging process (see preceding lines). We also tried to image several subresolution fluorescent beads but could not obtain sufficient signal when exciting at $\lambda=930 \mathrm{~nm}$ (not shown).

To get further insight into the nature of the DHE patches at the lower cell surface, we analyzed the intensity fluctuations per pixel from the acquired frames by N\&B analysis (Figs. 4E-4I). This method calculates the moments of the fluorescence intensity distribution for each pixel in an image time series (Digman et al., 2008). The apparent brightness, $B$, is given by the ratio of the second and first moment of the pixelwise intensity distribution [see Eq. (6)]. The N\&B analysis allows for discriminating pixels containing fluctuations from many dim molecules from those with fluctuations due to few bright molecules. Fluctuations being detectable as a statistical distribution of photon counts are caused by (1) the Poisson statistics of light emission and (2) by non-Poissonian intensity fluctuations due to traversal of the fluorescent molecules through the laser beam (Digman et al., 2008). The latter intensity fluctuations are either caused by a varying number or by varying brightness of fluorescent entities moving through the laser PSF. Few bright entities, like clusters of DHE, would cause large fluctuations resulting in large intensity variance associated with low mean intensity. This, in turn, would create high brightness $(B)$ values in those regions, where sterol domains would form [see Eqs. (6) and (7)]. Accordingly, only spatially varying brightness, coinciding with the calculated average image and $N$-map, would indicate sterol clusters. To test this hypothesis, we calculated first the apparent number of particles per pixel, $N$ (Fig. $4 \mathrm{E}$ ). The $N$-map was very similar to the image of average intensity (Fig. $4 \mathrm{G})$. As a consequence, the calculated brightness map, $B$, was homogeneous and contained no visible structures (Fig. 4F). The logic behind this conclusion can be seen by simple rearrangement of Eqs. (6) and (7), showing that the apparent brightness, $B$, can also be expressed as the ratio of average intensity and apparent number, $N$, in each pixel position (i.e., $B=\langle k\rangle / N$ ). Thus, the fact that for DHE average intensity and $N$-map look the same simply means that patches with higher average intensity contain more sterol molecules of the same brightness (i.e., no aggregates). Accordingly, intensity fluctuations around the mean value of the whole acquired image stack are spatially uniform, i.e., bright regions have proportionally the same fluctuations as dim regions (Figs. 4E-4G). An alternative explanation for this observation could be that DHE selfquenches in putative sterol clusters reducing the molecular brightness of these clusters to the value found for DHE monomers. According to this hypothesis, sterol clusters would exist, but they would not be brighter than individual sterol molecules. We can rule out this explanation for the following reasons: concentration self-quenching causes a lowered quantum yield being detectable by a slowed photobleaching decay of a flurophore (Benson et al., 1985; Hirschfeld, 1976). We showed recently that DHE's quantum yield and photobleaching behavior are spatially invariant in cellular membranes and that DHE does not self-quench up to $45 \mathrm{~mol} \%$ of the sterol in lipid bilayers (Garvik et al., 2008; Wüstner et al., 2010). Thus, any clustering of
DHE, i.e., association of DHE molecules, would result in local variation in molecular brightness, $\epsilon$, which would cause spots or structures in the $B$ map. These regions of large $B$ values would coincide with resolvable structures in the average intensity image, as recently demonstrated for aggregates of cell adhesion proteins in the plasma membrane (Digman et al., 2008). When plotting the calculated molecular brightness, $\epsilon$, against average intensity, we found no correlation between both parameters (Fig. $4 \mathrm{H}$ ). Together, statistical image analysis strongly indicates that areas of apparently high DHE intensity contain in fact more membrane but not clusters of the fluorescent sterol. A few dim spots in the brightness map might indicate vesicles transiently moving toward the plasma membrane, thereby appearing as one large entity containing DHE. This has been shown earlier for other cell types and fluorescent molecules (Digman et al., 2008). It is likely that vesicles contribute to the MP image shown in Figure 4, since the DOF of our MP setup of $\sim 0.8 \mu \mathrm{m}$ cannot distinguish plasma membrane patches from sterol-containing vesicles in close proximity to the cell surface.

To obtain spatiotemporal information about membrane dynamics, we performed time-lapse experiments, where a HepG2 cell pulse labeled with DHE was placed at a temperature-controlled microscope of the MP microscope maintained at $35 \pm 2{ }^{\circ} \mathrm{C}$ stage 5 min after staining (Fig. 5). Nine frames were recorded every 1 min with an acquisition time of 2 s/frame and with a laser power of $50 \mathrm{~mW}$. The sum projection of these nine frames was calculated to obtain one image with high SNR at every time point. Note that we focused at the perimeter of the cell for this experiment to distinguish between sterol membrane dynamics and movement of DHE-containing vesicles. For data analysis, we used our previously developed protocol (Wüstner, 2008; Wüstner and Færgeman, 2008b) and extracted intensity profiles along the plasma membrane over the complete time-lapse sequence (Figs. 5A and 5B). From the $1 \mathrm{D}$ intensity profiles, a separate spatial and temporal autocorrelation analysis was performed as described in Materials and Methods. The temporal correlation analysis revealed that about half of the DHE-containing surface structures are immobile over the course of the time-lapse experiment (Fig. 5C). This can be inferred from a monoexponential fit of the form $f(t)=a \cdot e^{-k \cdot t}+b$ to the normalized temporal correlation curve; the parameter $b$ describes the nondecaying fraction of the time correlation and indicates the contribution of immobile structures to the total temporal correlation coefficient. Accordingly, $R(0)$ of Eq. (5) equals the sum of the fit values for the parameters $a$ and $b$, while $b$ resembles the residual correlation $R(t \rightarrow \infty)$ using the monoexponential decay function stated earlier. The same analysis revealed a typical correlation time of $\tau=2.5 \mathrm{~min}$ for the mobile fraction. This value can be calculated from $\tau=\ln 2 / k$, with $k$ being the fitted decay rate constant in the monoexponential model. This is very close to the value we determined previously with the same type of analysis from WF timelapse sequences of DHE-stained cells (Wüstner and Færgeman, 2008b). In those experiments, we also observed a large immobile fraction of sterol in the plasma membrane [i.e., a monoexponential fit of the 
form given earlier shows that temporal correlation leveled off to a value of $R(t \rightarrow \infty)=b=0.77$ (Wüstner and Færgeman, 2008b)]. Note that the time resolution of this experiment is $\geq 1 \mathrm{~min}$, whereas that of the sequence used in the N\&B analysis (stated earlier) was much higher (around $2 \mathrm{~s}$ between frames). Thus, the experiment shown in Figure 5 was performed for assessing slower dynamic processes in the plasma membrane.

To get statistically meaningful data, this type of time-lapse experiment and the associated analysis (stated earlier) was performed on several cells $(n=8)$ giving a correlation time of $\tau=2.65 \pm 0.2 \mathrm{~min}$ and a residual value of $R(t \rightarrow \infty)=b=0.69 \pm 0.11$ (mean \pm standard deviation). Immobile components in the temporal correlation curve, as estimated by the residual $b$ in the fit, could be either a consequence of static sterolenriched domains in the plasma membrane or of the convolution of the cell surface with membrane folds being immobile over the course of our experiment (Wüstner, 2007b). We strongly prefer the latter explanation for the following reasons. First, we obtained very similar values in a fit of the monoexponential function to intensity profiles of cells labeled with the lipid probe DiIC12 in our previous study (Wüstner and Færgeman, 2008b). The lipid analog DiIC12 is a marker for the liquid-disordered phase in ternary model membrane lipid mixtures, where DiIC12 completely segregates from DHE, the latter preferring the liquid-ordered phase (Garvik et al., 2008). Thus, if the immobile structures observed for DHE in both MP (this study, Fig. 5) and WF-UV microscopy (Wüstner and Færgeman, 2008b) would resemble static sterolenriched domains, the fluid lipid marker DiIC12 should not show the same behavior. Second, by the N\&B analysis (stated earlier) shown in Figure 4, we came independently to the same conclusion, namely that static membrane convolutions cause an apparent enrichment of DHE in the plasma membrane of living cells.

The characteristic peak width in the smallest resolvable scale of the measured intensity line profiles gave a FWHM of $250 \mathrm{~nm}$ (Fig. 5E). This is in close agreement to the values we obtained at cell attachment sides (see preceding text and Figs. 4C and 4D). The spatial autocorrelation data contains nonperiodic fluctuations excluding the possibility of a fitting with a simple decay function (Fig. 5D; Tokumasu et al., 2005). Therefore, we only used spatial lag values up to $1 \mu \mathrm{m}$ to estimate the effective MP beam radius, $\omega_{\mathrm{mp}}$ (Fig. $5 \mathrm{~F}$ ). A halfsided Gaussian function of the form

$$
r(\xi, 0)_{t}=g(0,0)_{n} \cdot \exp \left(-\frac{\xi^{2}}{\omega_{\mathrm{mp}}^{2}}\right)+g_{\infty n}
$$

was fitted to the decaying autocorrelation curve [compare Eq. (4); Hebert et al. (2005b)]. Here, the parameters estimated from the nonlinear regression of this function to the decaying autocorrelation curve are the zero-lag autocorrelation amplitude, $g(0,0)_{n}$, the MP correlation radius $\omega_{\mathrm{mp}}$, which is proportional but not identical to the laser beam $e^{-2}$ lateral focus radius, and the offset $g_{\infty n}$. In fact, $\omega_{m p}$ is the effective MP beam radius, i.e., the focal laser region, which indeed excites DHE by a MP process (Wiseman et al., 2000). Nonlinear regression of this function to the experimentally determined autocorrelation curve gives a very good fit (Fig. $5 \mathrm{~F}$ ) and yields a value of $\omega_{\mathrm{mp}}=182 \pm 22 \mathrm{~nm}$, well in accordance with the published data (Hebert et al., 2005b; Schrader et al., 1997). Since the pixel size of our detector system is $\Delta x y=72.65 \mathrm{~nm}$, it follows that the effective MP beam radius $\omega_{\mathrm{mp}}$ equals $\sim 2.5$ pixels. Thus, the necessary conditions given by the Nyquist criterion and image correlation theory are fulfilled (Petersen et al., 1993). The MP beam radius, $\omega_{\mathrm{mp}}$, estimated by this analysis, was required to determine diffusion coefficients of sterol-containing vesicles, as described in the following.

\section{Measurement of Mobility of DHE-Containing Intracellular Vesicles by STICS}

In time-lapse sequences like the one shown in Figure 5 , we observed interesting vesicle dynamics. For example, a vesicle formed at the plasma membrane and moved into the cytoplasm over a time course of $5 \mathrm{~min}$, resembling sterol endocytosis (Fig. 6A, upper zoom box). Moreover, tiny vesicles with a typical diameter of 240-260 nm were found in the whole cytoplasm. Their sizes were inferred from the FWHMs of Gaussian fits to measured line profiles of individual vesicles (not shown, but see Fig. 4, above). An example for such a cytoplasmic vesicle is shown in the lower zoom box of Figure 6A; this vesicle occurred in the focal plane and stayed there for about $4 \mathrm{~min}$, before its intensity became weaker and slightly blurred, indicating movement out of the focus. In a similar MP time-lapse sequence, we observed stretching of vesicles during their intracellular movement, indicating transport along cytoskeleton tracks (Figs. $6 \mathrm{~B}$ and $6 \mathrm{~B}^{\prime}$ ). Such shape changes of DHE-containing vesicles cannot be resolved by UV-WF microscopy due to (i) higher out-offocus fluorescence resulting in blurred vesicle images and (ii) more rapid photobleaching of the fluorescent sterol and thus lower SNR. Longer tracking was impossible since most vesicles disappeared probably because they moved out of the focal plane. Note again that the DOF of MP microscopy is very narrow (i.e., $\sim 0.8 \mu \mathrm{m}$ ) compared to that of UV-WF microscopy, where individual vesicles can be identified over a distance of more than $8 \mu \mathrm{m}$, even when increasingly blurred (Wüstner and Færgeman, 2008b).

To illustrate that the pattern of DHE-containing vesicles changes significantly over time, a color overlay was made with the start image $(t=0 \mathrm{~min})$ shown in green and the red image representing either the second frame $\left(t=1 \mathrm{~min}\right.$; Figs. $6 \mathrm{C}$ and $\left.6 \mathrm{C}^{\prime}\right)$ or the tenth frame $\left(t=9 \mathrm{~min}\right.$; Figs. $6 \mathrm{D}$ and $\left.6 \mathrm{D}^{\prime}\right)$. The ability of correlating fluorescence intensity fluctuations with the scanning laser beam in the MP microscope should allow for obtaining new insights into intracellular dynamics of DHE (Kolin and Wiseman, 2007). We decided to use STICS to obtain information about the dynamics of these sterol-containing vesicles (Fig. 6E). This technique allows for measuring diffusion constants of particle movement from the decay of the temporal correlation at $r(0,0)$ and provides additionally information about the presence, direction, and velocity of eventual flow components, indicated by changing position of the correlation peak (Brown et al., 2006; Hebert et al., 


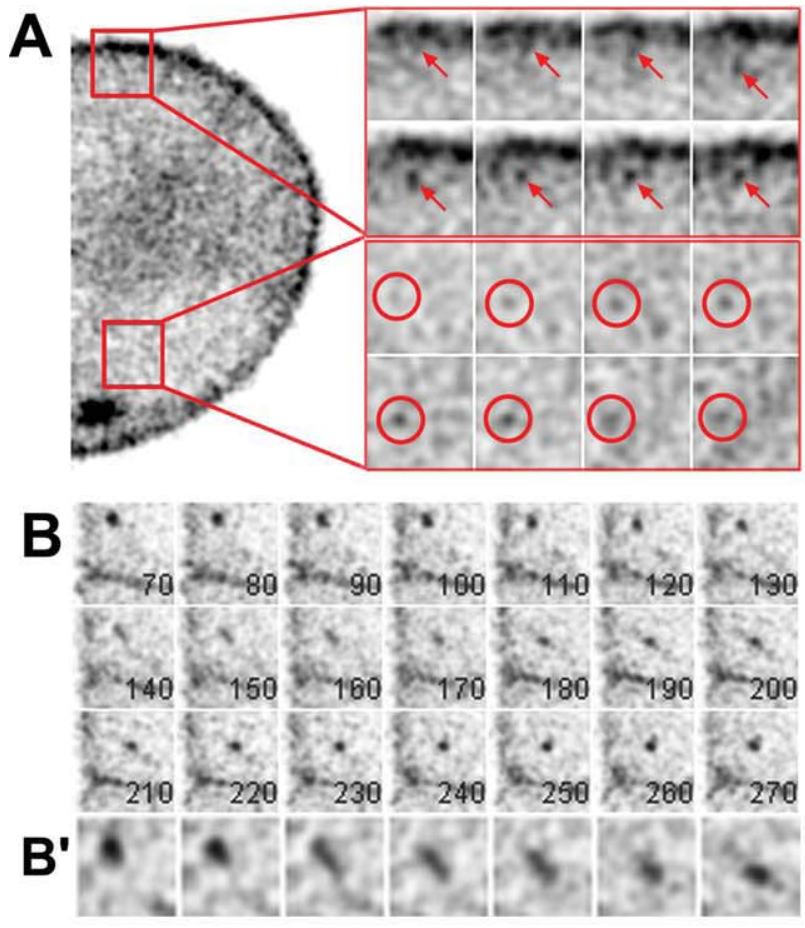

Fig. 6. MP imaging of sterol transport in cytoplasmic vesicles. HepG 2 cells were labeled with DHE/MCD for 1 min, washed, chased for $30 \mathrm{~min}$ at $37^{\circ} \mathrm{C}$, and placed on the temperature-controlled stage of a MP microscope maintained at $35 \pm 1^{\circ} \mathrm{C}$. The perimeter of the cell was imaged over a time course of $25 \mathrm{~min}$ with nine averaged frames recorded every $1 \mathrm{~min}$ at $37^{\circ} \mathrm{C}$ (see Fig. 5). A: Representative inverted image of the cell perimeter with two zoom boxes. Upper box shows the event of formation of a DHE-containing vesicle at the plasma membrane. Lower zoom box shows appearance and disappearance of a sterol-containing cytoplasmic vesicle in the focal plane during the time-lapse recording. B: Zoomed region from another time lapse dem]-

2005a; Kolin and Wiseman, 2007). The fact that there was no change in the position of the Gaussian peak of the spatiotemporal autocorrelation in our experiment shows that no cytoplasmic flow or streaming was present in this sample (Fig. 6E; Hebert et al., 2005a). Thus, mobility of DHE-containing vesicles can only be caused by diffusion, maybe interrupted by short episodes of directed movement along cytoskeleton elements. We calculated the spatiotemporal autocorrelation for several such time-lapse sequences (Fig. 6E) and performed a fit of Eq. (3) to the temporal correlation function $r(0,0, \tau)$. This function was inferred from the zero-spatial-lag peak of the respective spatiotemporal autocorrelation function using tools in ImageJ (Hebert et al., 2005a). With the experimentally determined laser correlation radius of $\omega_{\mathrm{mp}}=182 \mathrm{~nm}$ (see preceding text, Fig. $5 \mathrm{~F}$ ), the fit gave a $2 \mathrm{D}$ diffusion coefficient of $D=$ $0.501 \pm 0.045 \times 10^{-13} \mathrm{~cm}^{2} / \mathrm{s}$ (Fig. 6E). This value is by a factor of 20 lower than that we determined previously by UV-WF time-lapse imaging and particle tracking of DHE-containing vesicles in J774 macrophages (Wüstner and Færgeman, 2008b). By comparing both diffusion constants, one has to take into account that

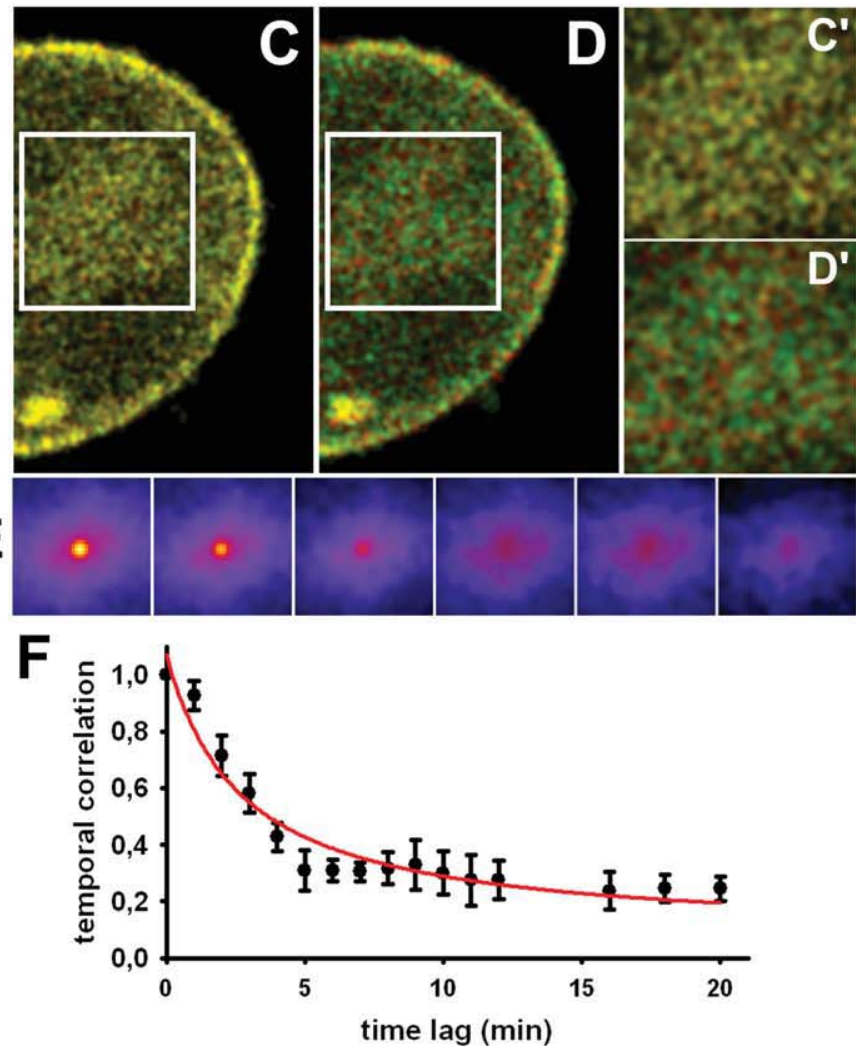

onstrating displacement of a cytoplasmic vesicle containing DHE accompanied by a change of vesicle shape. $\mathbf{B}^{\prime}$ : Zoom of the frames indicated by " 120 " to " 180 " showing the elongation of vesicle shape in direction of vesicle movement. C, D: Color overlay of the whole field, as shown in (A), for the start image $(t=0$, green) and the second image $\left(t=1 \mathrm{~min}, \mathrm{red}, \mathrm{C}, \mathrm{C}^{\prime}\right)$ or tenth image $\left(t=9 \mathrm{~min}, \mathrm{red}, \mathrm{D}, \mathrm{D}^{\prime}\right)$. E: Spatiotemporal autocorrelation images for the first six time points (including $t=0)$. F: Temporal autocorrelation determined from a line profile through the peak intensity of the autocorrelation images as shown in (E) but for eight different time-lapse sequences (mean \pm SEM). Black symbols, data, red line fit to Eq. (3). See text for further explanations.

different cell lines were used in this and our previous study and that different imaging modalities and evaluation strategies were applied. In nonpolarized HepG2 cells, like those used in the experiment shown in Figure 6 , we observed often rather stationary vesicles by UV-WF time-lapse microscopy (Wüstner, 2005, 2007a; Wüstner et al., 2002). Moreover, the quality of the fit of Eq. (3) to the correlation function in Figure $6 \mathrm{E}$ is far from being perfect. In fact, we obtained a much better fit using a compressed exponential function (see Supporting Information Fig. S2). This function can account for the delayed decay, which we observed in the data (see Fig. 6E).

\section{Potential of MP Microscopy and UV-WF Imaging for Colocalization Studies of DHE}

Further characterization of sterol transport by fluorescence microscopy requires to identify target compartments by costaining organelles with specific markers, e.g., enhanced green fluorescent protein (EGFP)-tagged proteins. In a recent study, we demonstrated by UV-WF microscopy that DHE colocalizes with EGFP-tagged Niemann Pick C1-like protein1 
(EGFP-NPC1L1) in the ERC and that this protein enhances sterol transport to the recycling endosomes (Hartwig Petersen et al., 2008). Using this system, here, we tried to acquire images of DHE and EGFPNPC1L1 simultaneously in our MP microscope using a laser wavelength of $930 \mathrm{~nm}$. At this wavelength, DHE and EGFP-NPC1L1 become simultaneously excited, probably by a three-photon and two-photon process, respectively. Parallel excitation and imaging, however, gave poor results for the EGFP-tagged protein due to excitation saturation of the fluorophore (not shown). This is a consequence of the extremely high laser power being required to excite DHE (Zipfel et al., 2003). Thus, we were urged to acquire both images sequentially, with reduced laser power for imaging EGFP-NPC1L1 (0.5 mW) compared to DHE (50 mW). By this approach, colocalization of DHE with EGFPNPC1L1 was found in the plasma membrane, the ERC, and a few peripheral endosomes (Figs. 7A-7C). For three-color experiments, with, e.g., Alexa546-Tf, we would need to get excitation wavelengths of $>1,000$ nm, currently not available with our MP setup. For comparison, cells expressing EGFP-NPC1L1 were colabeled with DHE and Alexa546-Tf, and colocalization between all three channels was determined by UVWF microscopy. Colocalization in UV-WF imaging modus requires correction for chromatic aberration between the UV and green or red channel providing good-quality images of colocalizing species (Figs. 7D7H; Wüstner and Færgeman, 2008a). Together, colocalization of DHE with EGFP-NPC1L1 can be detected by both methods. A major limitation of MP microscopy colocalization studies with DHE is the low time resolution: with our setup about 1 min lye between acquisition of both images allowing for reasonable movement of labeled vesicles. This could be wrongly interpreted as segregation of both probes (see ERC region in zoomed panel in Fig. $7 \mathrm{C}^{\prime}$ ). In contrast, with a wellaligned UV-WF microscope, we were able to reduce the time between image acquisitions in two channels including adjusting the focus position to less than $6 \mathrm{~s}$. The ease implementation of dual and even triple-color imaging in UV-WF modus suggests that this imaging method is better suited for colocalization studies than MP microscopy of DHE-labeled specimen.

\section{Improved SNR of DHE in Cells by UV-WF Bleach-Rate Fitting}

The more efficient excitation of DHE in UV-WF microscopy compared to MP microscopy is of great promise for future studies, if one could overcome the signal degradation by rapid bleaching of DHE in this imaging mode. We tried previously to circumvent this problem in two steps: (1) acquiring a bleach stack by repeated acquisition of DHE-stained specimen and fitting a monoexponential decay function to the fading DHE intensity over the whole image field followed by (2) multiplying the images in a postprocessing step by the reciprocal of this decay function, eventually followed by frame averaging (Markham and Conchello, 2001; Wüstner, 2005; Wüstner and Færgeman, 2008a). This method allowed for some gain in SNR and for applying deconvolution algorithms to small $z$-stacks of DHEstained cells acquired on a UV-WF microscope system (see Fig. 3G and Wüstner, 2005; Wüstner and Færge-
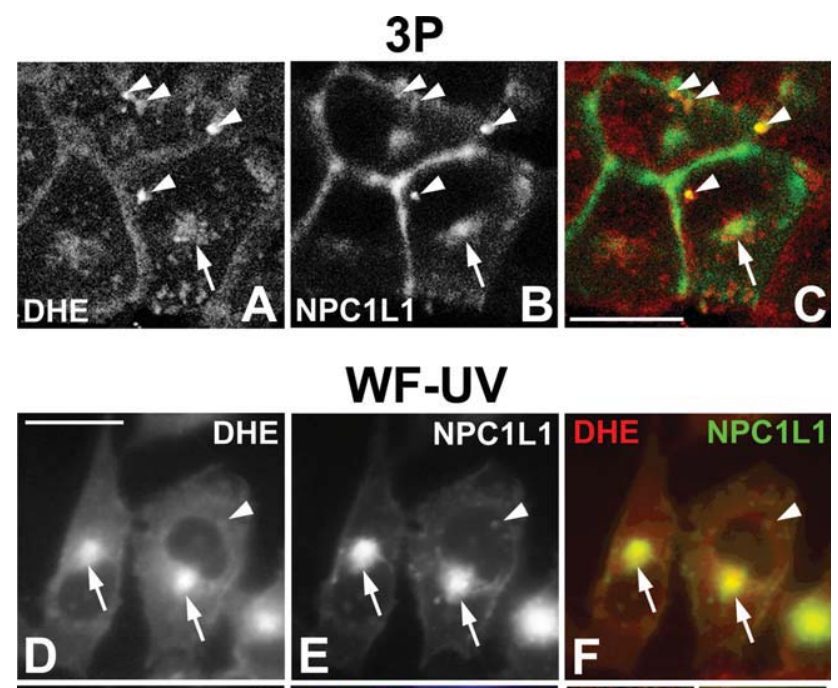

WF-UV
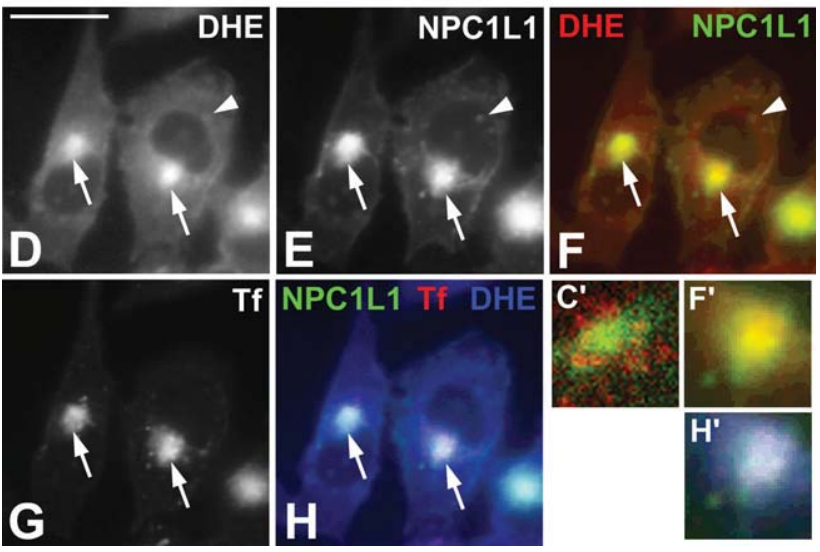

Fig. 7. Colocalization of DHE with EGFP-tagged NPC1L1 (EGFPNPC1L1) studied by MP and UV-WF microscopy. A-C: McA cells expressing EGFP-NPC1L1 were labeled with DHE/MCD for $1 \mathrm{~min}$, washed, chased for $30 \mathrm{~min}$ at $37^{\circ} \mathrm{C}$, and imaged on a MP microscope using one excitation wavelength $(930 \mathrm{~nm})$ for both probes with sequential scanning of both channels. D-H: McA cells expressing EGFP-NPC1L1 were labeled with DHE/MCD for 1 min, washed, and chased for $30 \mathrm{~min}$ at $37^{\circ} \mathrm{C}$ in the presence of $20 \mu \mathrm{g} / \mathrm{mL}$ Alexa546-Tf. Cells were washed and imaged on a UV-WF microscope. A, D: DHE; B, E: EGFP-NPC1L1; G: Alexa546-Tf; C, C' , F, F': Color overlay with DHE in red and EGFP-NPC1L1 in green. Color overlay with DHE in blue, EGFP-NPC1L1 in green, and Alexa546-Tf in red $\left(\mathrm{H}, \mathrm{H}^{\prime}\right)$. Arrows point to the perinuclear ERC, whereas arrowheads indicate peripheral vesicles containing sterol and NPC1L1. Bar, $20 \mu \mathrm{m}$.

man, 2008a). Since noise is also multiplied by the correction procedure, the overall gain in image quality, however, is limited (Wüstner and Færgeman, 2008a). An alternative approach to improve image quality in UV-WF imaging of DHE would be to gather the whole information contained in the bleach stack by considering the bleach process pixelwise. We introduced recently a new image segmentation algorithm suitable for detecting DHE in vivo in the presence of high autofluorescence (Wüstner et al., 2010). In this method, implemented as plugins to the popular image analysis software ImageJ and called PixBleach (available at http://bigwww.epfl.ch/algorithms/pixbleach), a user-definable decay model is fitted pixelwise to DHE bleaching in an image stack. For example, in case of monoexponential bleaching, as found for DHE in model membranes, cells, and living nematodes, one obtains from the fit an amplitude image, a time constant image, and a background image (Wüstner et al., 2010). Moreover, the algorithm estimates the RMS deviation (RMSE) and chi-square values of the goodness of fit and calculates a reconstructed bleach stack based on the estimated parameters. As shown in Figure 8A for DHE-labeled HepG2 cells, the reconstructed ("fit") and raw 

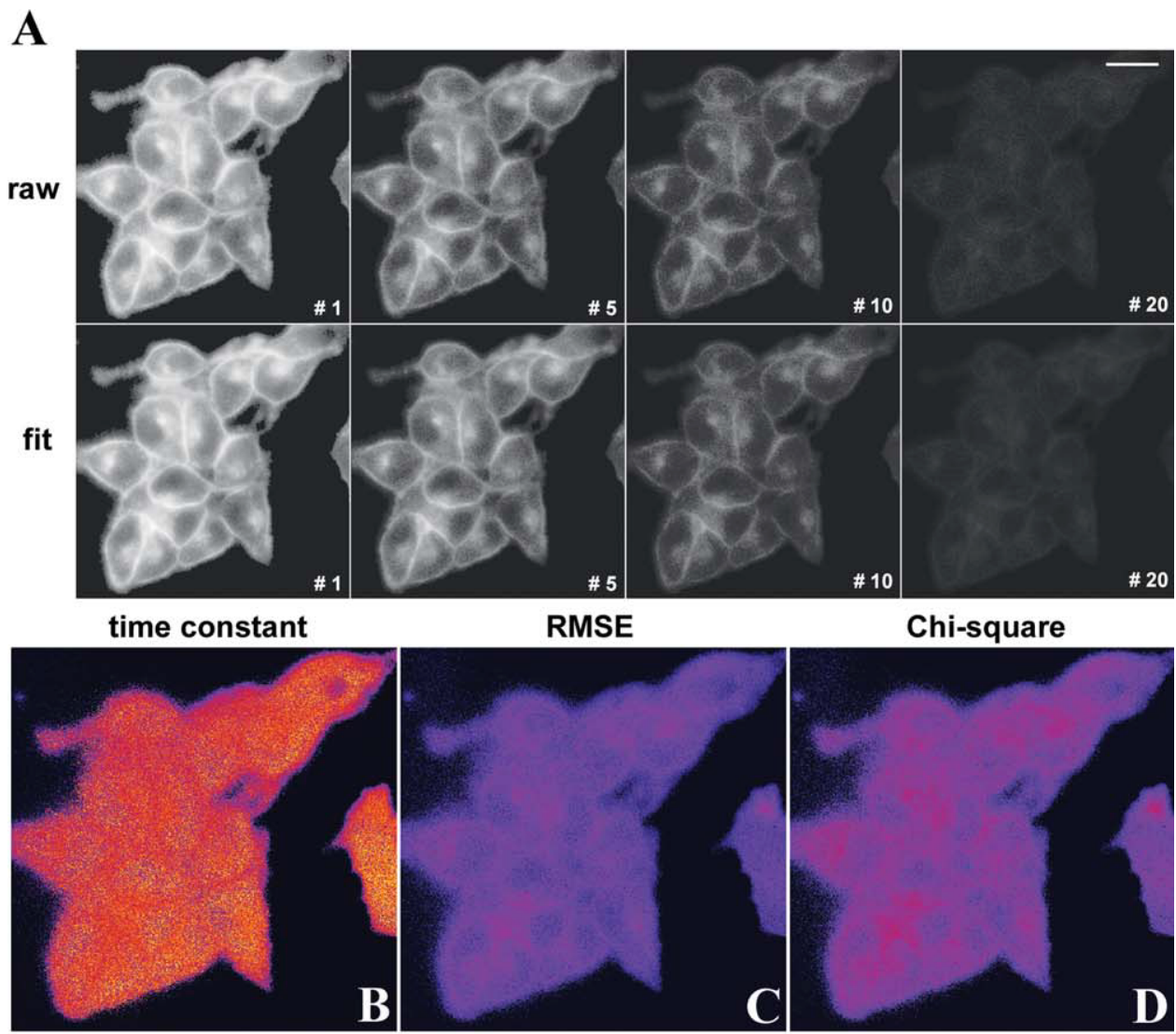

RMSE

\section{Chi-square}

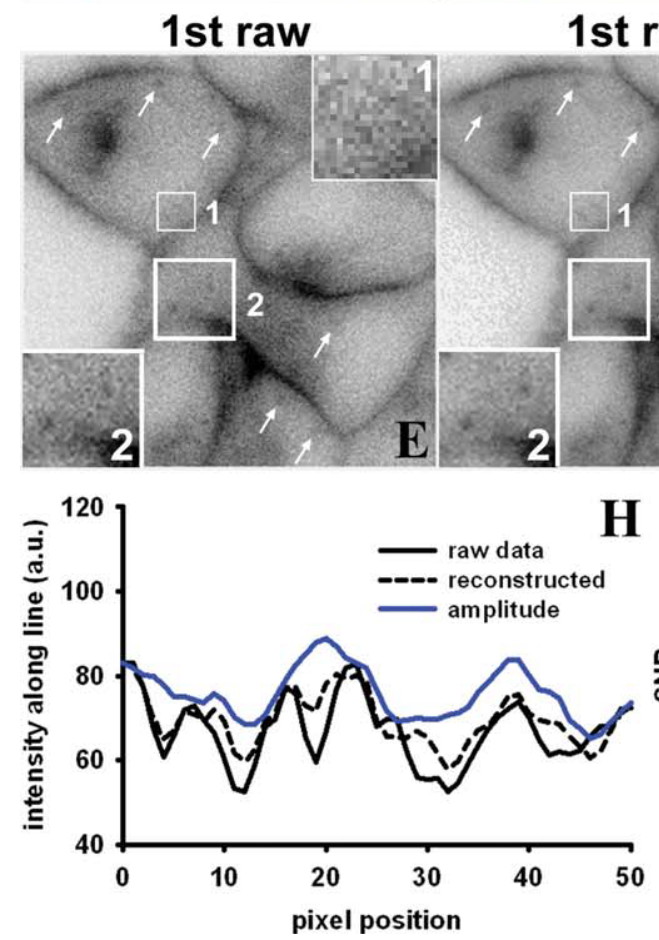

rec.

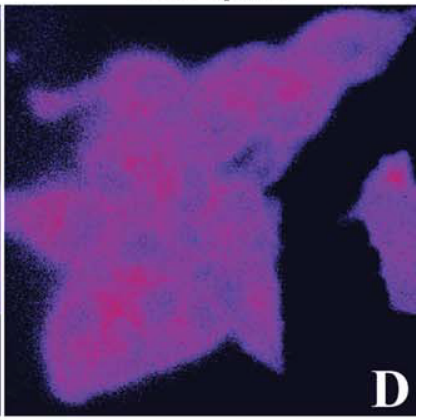

amplitude
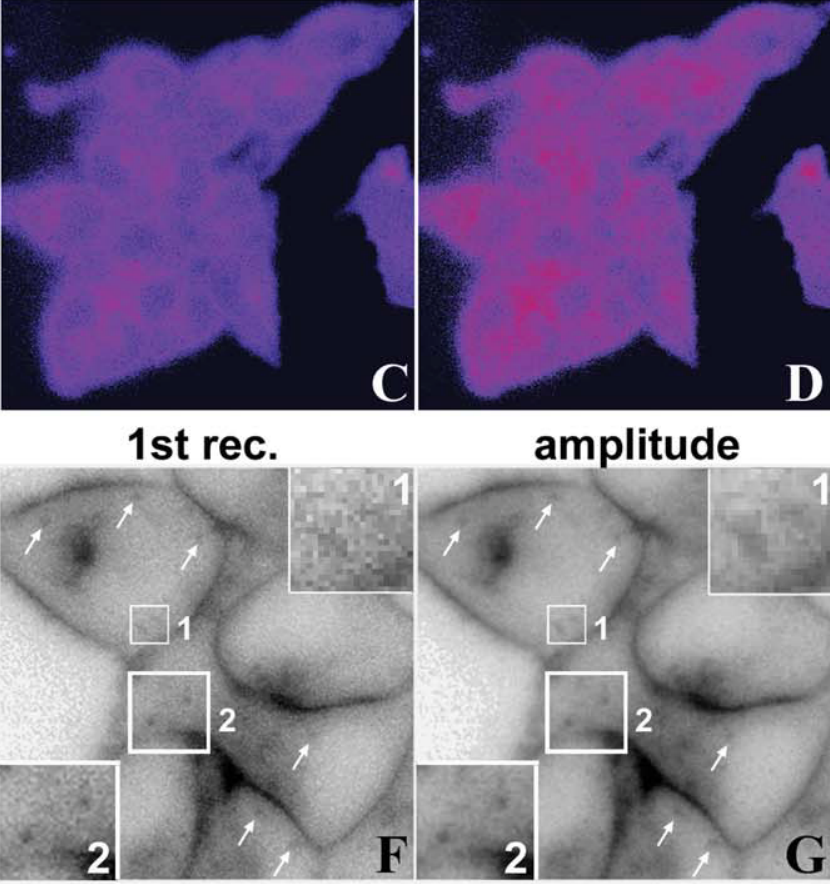

$\square 1$
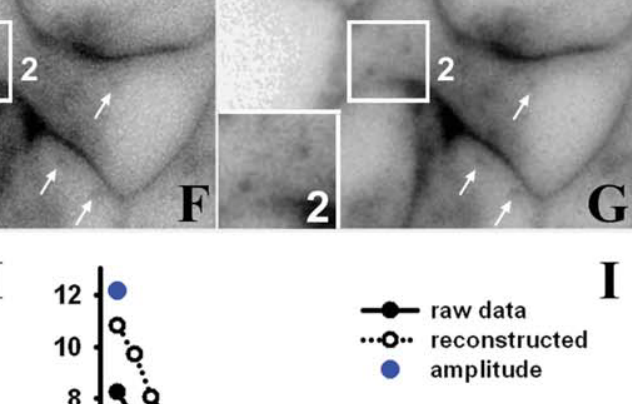

Fig. 8. Improvement of SNR in UV-WF imaging by pixelwise bleach rate fitting. HepG2 cells were labeled with DHE/MCD for 1 min, washed, chased for $30 \mathrm{~min}$ at $37^{\circ} \mathrm{C}$, and imaged on a UV-WF microscope with repeated acquisition of the same field resulting in strong photobleaching (A, top, "raw"). This bleach stack was fitted pixelwise to a monoexponential decay function using PixBleach, a plugin to ImageJ previously developed by us (Wüstner et al., 2010). A, bottom, "fit": The reconstructed stack is shown. Bar, $15 \mu \mathrm{m}$. B: Time constant; C: RMS (RMSE); D: Chi-square map. E-G: Comparison of the first image of the original bleach stack (E, "1st raw") and of the

reconstructed bleach stack (F, "1st rec.") compared to the amplitude image of the fit $(\mathrm{G})$. Zoomed boxes show improved image quality in the reconstructed and amplitude image, respectively. Images shown are inverted for presentation purposes. $\mathbf{H}$ : Line scan across the two DHE-containing structures of box 1 for the raw data (black straight line), the reconstructed data (black dashed line), and the amplitude image (blue straight line). I: Calculated SNR ratio for the raw data (black circles), the reconstructed data (white circles), and the amplitude image (blue circle). See text for further explanations. 
data ("raw") are almost identical, whereas the time constant map confirms spatially invariant bleaching of DHE (Fig. 8B). Importantly, we observed that in case of good fit quality (i.e., low RMSE and chi-square values), the reconstructed images are of better quality than the raw data. Moreover, the amplitude image estimated from fitting the monoexponential decay model to the bleach stack has even better contrast because of suppression of image noise (compare Figs. 8E-8G). Small vesicles contained already in the raw data (see first acquired plane of the bleach stack in Fig. 8E) are better discernable in the reconstructed (Fig. 8F) and even better in the amplitude image (Fig. 8G). This is exemplified within the two zoomed box regions and in the line profile across the small vesicular structures from box 1 (compare Figs. 8E-8G and Fig. 8H). We estimated the gain in SNR as function of acquired frames as explained in Materials and Methods and observed a most pronounced improvement in SNR for the first two to three frames when comparing reconstructed and raw data image stacks (Fig. 8I). The calculated SNR is even higher in the amplitude map $(\mathrm{SNR}=12.14)$ than in the first plane of the reconstructed image stack $(\mathrm{SNR}=10.80)$, which is again higher than that in the first frame of the raw data (SNR = 8.26; Fig. 8I). Thus, the SNR estimated independently for each data set confirms the visual impression of better image quality in the reconstructed data. We explain this interesting result by the fact that some image noise, contained in the raw data, is removed in the reconstructed data and the amplitude map, simply because noise does not bleach. Accordingly, contrast of small DHE-containing structures with intensities hardly above image noise in the raw data is enhanced in the reconstructed image and even more pronounced in the amplitude map. Thus, for low-light-level applications, such as DHEstained specimen with a rapidly bleaching fluorophore, gathering the whole information hided in the bleach stack has the potential to improve image quality. Cytoplasmic DHE-containing vesicles could be distinguished in the UV-WF bleach amplitude map due to improved contrast compared to the raw data. This result confirms experiments presented earlier for MP microscopy (see Fig. 6), namely, that tiny vesicles contribute to intracellular sterol transport in living cells.

\section{Comparison of Frame Averaging in UV-WF and MP Microscopy of DHE}

To assess the potential of frame averaging for UVWF imaging of DHE, we performed an additive correction for photobleaching in this imaging modality on a pixel-by-pixel basis [see Eq. (8) and Fig. 9]. Additive correction avoids multiplying noise and can account for the intensity fading as observed by repeated imaging of DHE (compare Fig. 9A with Fig. 8A and see Fig. 9J). Successive frame averaging of the raw and bleach-corrected stack gave very different results: averaging the raw image stack was accompanied by decreasing intensity (Fig. 9B, upper row), while this was not found for the bleach-corrected data (Fig. 9B, lower row). By comparing the amplitude image (Fig. 9C) with frame-averaged $(n=28)$ bleach-corrected (Fig. 9D) and raw image (Fig. 9E), two aspects become apparent. First, content and quality of amplitude and frame-averaged bleachcorrected image are comparable (Figs. 9C and 9D). Sec- ond, the frame-averaged raw image deviates significantly from the amplitude image (Fig. 9E, compare Fig. 9C). This is confirmed by the PSNR calculated between amplitude and frame-averaged bleach-corrected and frame-averaged raw image (Figs. 9D and 9E, respectively). A principal component analysis (PCA) confirms this conclusion and shows that the strongest deviation between amplitude and frameaveraged bleach-corrected image on one side and frame-averaged raw image on the other side is found in the second principle component [PC2; Fig. 9G; see Wüstner (2007b) for details and calculation of PCA]. We also performed a colocalization analysis and found that the Pearson coefficient, $R_{\mathrm{p}}$, measuring correlation between two image channels, was lower for amplitude versus frame-averaged raw image than for amplitude and frame-averaged bleach-corrected image (Fig. 9I). Careful inspection of the color overlays revealed that in the frame-averaged raw image new structures appear (inset, color panels in Fig. 9I). These tubule-like structures were not found in the amplitude image or the frame-averaged bleach-corrected image (compare also Figs. 9C-9E). They were neither observed in the first image of the original bleach stack, suggesting that these tubules do not contain any DHE. They are likely autofluorescent structures, as typically observed in the last frames of a DHE bleach stack. We demonstrated previously that cellular autofluorescence in the UV region of the spectrum bleaches much slower than DHE and that extending the acquisition time samples mostly the autofluorescence (Wüstner et al., 2010). Accordingly, autofluorescence is estimated as background term $B(x, y)$ in our pixelwise bleach rate fitting procedure [see Eq. (8) and Wüstner et al. (2010)]. Indeed, the nonbleaching fraction of the UV-WF image stack shown in Figures 8 and 9 looks very much like the last frame of the raw image stack and is very similar to the second principle component shown in Figure 9G (see Supporting Information Fig. S3). From the results presented in Figure 9, one can conclude that frame averaging of the raw image stack strongly overemphasizes autofluorescent structures. This was not observed when image stacks were corrected for photobleaching using Eq. (8) before frame averaging. On the basis of these results, we wanted to determine whether frame averaging of bleach-corrected UV-WF stacks of DHE-stained cells will result in improved image quality relative to the amplitude image of the pixelwise fit. The PSNR as a relative measure of image quality was calculated between amplitude image as reference and successive frame averages of the bleach-corrected and raw image stack, respectively (Fig. 9K). We found that the measured PSNR leveled off after five averaged frames in case of the bleach-corrected stack (Fig. 9K, open symbols). Averaging five frames of the raw image stack gave an even better PSNR than averages of five bleach-corrected frames (Fig. 9K, closed symbols). This might be due to slightly less noise in the first five frames of the raw image stack compared to the bleachcorrected stack because some noise is added by the bleach correction procedure [see Eq. (8)]. However, averaging more than five frames resulted in a steep decline in the PSNR for the raw image stack but not for the bleach-corrected stack (Fig. 9K, compare open and closed symbols). We take this as evidence for 

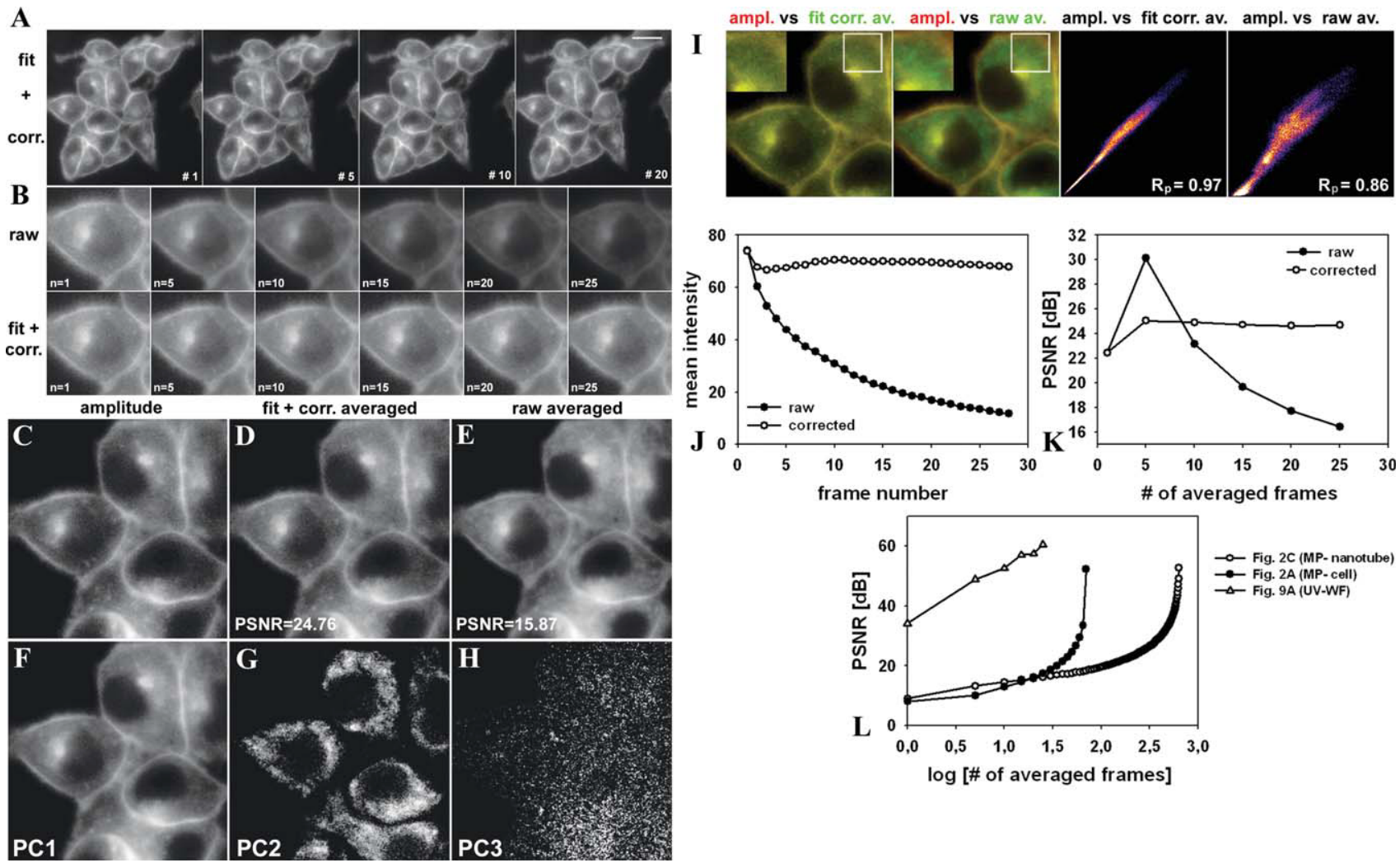

Fig. 9. Frame-averaging and pixelwise bleaching correction. The same data set as shown in Figure 8 was first corrected for bleaching on a pixel-by-pixel basis using the parameters obtain by the bleach-fit and Eq. (8) (see Materials and Methods). A: Example frames (number indicated) of the bleach-corrected stack ("fit + corr."). Bar, $15 \mu \mathrm{m}$. B: Successive frame averages (number of frames entering the average is indicated) for the raw image stack (top) and the bleach-corrected stack (bottom). C: Amplitude of the bleach fit; D, E: Average of all 28 frames of the bleach-corrected (D) and raw image stack (E). The number on the image indicates the calculated PSNR relative to the amplitude image shown in (C). F-H: PCA for the images shown in (C-E). To get the same intensity population for this calculation, all three images were first normalized to their respective maximal value such that all intensities lie between 0 and 1. I (left two panels): Color overlay of a zoomed region of (C) in red (amplitude image) and (D) (bleach-corrected frame-averaged image) or (E) (raw frame-averaged image) in green, respectively. J: Mean intensity for the ROI shown in $(\mathrm{C}-\mathrm{E})$ for

the raw (closed symbols) and bleach-corrected stack (open symbols) as function of frame number (i.e., number of acquisitions). K: PSNR between amplitude image of the fit $(\mathrm{C})$ and successive frame averages of the raw (closed symbols) and bleach-corrected stack (open symbols) as function of number of averaged frames. L: The PSNR was calculated between last average (i.e., of the whole stack) and successive frame averages as function of the decadic logarithm of the number of averaged frames for the bleach-corrected UV-WF sequence (open triangles, compare A) and for the MP image sequences shown in Figure 2 (circles). PSNR for acquired sequences of Figure 2C (nanotube), recorded with $20 \mathrm{~mW}$ excitation power (open symbols), and of Figure $2 \mathrm{~A}$ (cell), recorded with $60 \mathrm{~mW}$ excitation power (closed symbols), relative to the total average of the sequence. This total average consisted of $n=28$ in case of UV-WF imaging and of $n=71$ and $n=650$ in case of MP microscopy for the image shown in Figures $2 \mathrm{~A}$ and $2 \mathrm{C}$, respectively. See text for further explanations.

increasing impact of autofluorescence on the averaged signal in the raw image stacks.

Finally, we compared the effect of extended frame averaging on image quality in UV-WF imaging with that in MP microscopy of DHE, the latter acquired with either low or high laser power (compare Figs. 2A and $2 \mathrm{C}$ and Fig. 9L). As reference image, we chose the average over all frames, for both the acquired bleachcorrected UV-WF stack $(n=28)$ and the unprocessed MP sequences ( $n=71$ and $n=650$; Fig. 9L). In case of MP imaging of DHE, longer frame averaging provides continuously better image quality (Fig. 9L, circles). The excitation laser power has only an impact on the number of averages being necessary to obtain a decent image quality. In other words, acquiring MP images of DHE with low laser power requires averaging more frames to get a certain PSNR between the total average and the sequence of frame averages. For example,

with high laser power $(60 \mathrm{~mW})$, a PSNR $>50$ is reached after averaging 70 acquisitions, whereas for a sequence acquired with low laser power $(20 \mathrm{~mW})$, more than 600 frames need to enter the average to receive the same PSNR (compare Figs. 2A and $2 \mathrm{C}$ and closed and open circles in Fig. 9L). A single acquisition was of very low quality for low and high laser power (PSNR < 10). In contrast, in case of UV-WF microscopy of DHE, already the first acquisition gives a PSNR $=34.165$, while averaging of 25 bleach-corrected frames resulted in image quality exceeding that of the acquired MP sequences (PSNR $=60.565$, triangles in Fig. 9L). Together, this analysis underlines the fundamentally different acquisition process in UV-WF and MP microscopy of DHE in cells. It has to be noted that calculation of PSNR is a relative measure of restoration quality because it is only valid within one image sequence (Gonzalez and Woods, 2002). Further improvement in 
image quality after frame averaging might be possible by implementing advanced denoising routines in both UV-WF and MP microscopy of DHE (Luisier et al., 2010).

\section{CONCLUSIONS}

Our study shows that UV-WF and MP excitation microscopy of DHE provide complementary information regarding intracellular sterol transport. While UV-WF imaging of DHE is fast and in combination with (limited) $z$-stacking and deconvolution suitable to perform multicolor colocalization studies of DHE, MP microscopy allows for obtaining $3 \mathrm{D}$ stacks with high resolution along the optical axis and time-lapse sequences with a very narrow DOF. These capabilities of MP microscopy are due to the intrinsic section effect and thereby reduced photobleaching limited to the narrow focal plane in this method. Moreover, we found that MP microscopy is able to resolve structural details below the lateral resolution limit of our UV-WF system; vesicles and plasma membrane patches containing DHE with a diameter of 210-250 $\mathrm{nm}$ could be resolved by MP microscopy (see Figs. 4-6), which is slightly smaller than the lateral resolution limit of our WF system. These structures have to remain at the same location, however, during the whole acquisition process because they otherwise become blurred. The fact that MP microscopy is a form of laser scanning microscopy allows for applying image correlation spectroscopy techniques, where spatiotemporal fluctuations in fluorescence intensity become correlated with the scanning laser beam. This capability provides new insights into membrane sterol distribution and intracellular sterol dynamics and is not available in UV-WF imaging of DHE. The requirement of extreme laser powers and rather sophisticated instrumentation, however, make MP microscopy not applicable for routine investigation of DHE transport, including multicolor applications. MP microscopy of DHE should therefore be used for rather specific questions as supplement to the wellestablished UV-WF imaging of intrinsically fluorescent sterols like DHE (Diaz et al., 2008; Hao et al., 2002; Hartwig Petersen et al., 2008; Hölttä-Vuori et al., 2005; Mondal et al., 2009; Mukherjee et al., 1998; Pipalia et al., 2006; Wüstner, 2007b, 2008; Wüstner and Færgeman, 2008a,b; Wüstner et al., 2002, 2004, 2005). For example, MP microscopy is an interesting alternative for in vivo sterol imaging because of its sectioning capability and deeper tissue penetration as shown in our most recent work (Wüstner et al., 2010). The techniques presented here might be of use for other applications of live-cell ultraviolet microscopy, like calcium imaging, monitoring cell metabolism using $\mathrm{NADH}$, or visualization of pyrene-tagged lipid probes.

\section{ACKNOWLEDGMENTS}

The authors thank Drs. Jay Unruh and Paul Wiseman very much for helpful discussions on image correlation spectroscopy and for providing plugins to ImageJ or MatLab routines for image correlation analysis, respectively.

\section{REFERENCES}

Aït-Slimane T, Galmes R, Trugnan G, Maurice M. 2009. Basolateral internalization of GPI-anchored proteins occurs via a clathrin- independent flotillin-dependent pathway in polarized hepatic cells. Mol Biol Cell 20:3792-3800.

Benson DM, Bryan J, Plant AL, Gotto AMJ, Smith LC. 1985. Digital imaging fluorescence microscopy: Spatial heterogeneity of photobleaching rate constants in individual cells. J Cell Biol 100:13091323.

Brown CM, Hebert B, Kolin DL, Zareno J, Whitmore L, Horwitz AR, Wiseman PW. 2006. Probing the integrin-actin linkage using highresolution protein velocity mapping. J Cell Sci 119:5204-5214.

Diaz G, Batetta B, Sanna F, Uda S, Reali C, Angius F, Melis M, Falchi AM. 2008. Lipid droplet changes in proliferating and quiescent 3T3 fibroblasts. Histochem Cell Biol 129:611-621.

Digman MA, Dalal R, Horwitz AF, Gratton E. 2008. Mapping the number of molecules and brightness in the laser scanning microscope. Biophys J 94:2320-2332.

Frolov A, Petrescu A, Atshaves BP, So PT, Gratton E, Serrero G, Schroeder F. 2000. High density lipoprotein-mediated cholesterol uptake and targeting to lipid droplets in intact L-cell fibroblasts. J Biol Chem 275:12769-12780.

Garvik O, Benediktsen P, Ipsen JH, Simonsen AC, Wüstner D. 2008. The fluorescent cholesterol analog dehydroergosterol induces liquid-ordered domains in model membranes. Chem Phys Lipids 159:114-118.

Gonzalez RC, Woods RE. 2002. Digital image processing. Upper Saddle River, NJ: Prentice Hall. Chapter 11, pp. 675-683.

Hao M, Lin SX, Karylowski OJ, Wüstner D, McGraw TE, Maxfield FR. 2002. Vesicular and non-vesicular sterol transport in living cells. The endocytic recycling compartment is a major sterol storage organelle. J Biol Chem 277:609-617.

Hartwig Petersen N, Færgeman NJ, Yu L, Wüstner D. 2008. Kinetic imaging of NPC1L1 and sterol trafficking between plasma membrane and recycling endosomes in hepatoma cells. J Lipid Res 49:2023-2037.

Hebert B, Costantino S, Wiseman PW. 2005a. Spatiotemporal image correlation spectroscopy (STICS) theory, verification, and application to protein velocity mapping in living $\mathrm{CHO}$ cells. Biophys $\mathrm{J}$ 88:3601-3614.

Hebert B, Hulme SE, Wiseman PW. 2005b. Membrane protein dynamics measured by two-photon ring correlation spectroscopy: Theory and application to living cells. In: Periasamy A, So PT, editors.Multiphoton microscopy in the biomedical sciences V. Proceedings of the SPIE, Vol. 5700. Bellingham, WA: SPIE. pp. 109-117.

Hirschfeld T. 1976. Quantum efficiency independence of the time integrated emission from a fluorescent molecule. Appl Opt 15:31353139

Hölttä-Vuori M, Alpy F, Tanhuanpaa K, Jokitalo E, Mutka AL, Ikonen E. 2005. MLN64 is involved in actin-mediated dynamics of late endocytic organelles. Mol Biol Cell 16:3873-3886.

Kolin DL, Wiseman PW. 2007. Advances in image correlation spectroscopy: Measuring number densities, aggregation states, and dynamics of fluorescently labeled macromolecules in cells. Cell Biochem Biophys 49:141-164.

Kolin DL, Costantino S, Wiseman PW. 2006. Sampling effects, noise, and photobleaching in temporal image correlation spectroscopy. Biophys J 90:628-639.

Luisier F, Vonesch C, Blu T, Unser M. 2010. Fast interscale wavelet denoising of Poisson-corrupted images. Signal Processing 90:415427.

Markham J, Conchello JA. 2001. Artefacts in restored images due to intensity loss in three-dimensional fluorescence microscopy. J Microsc 204:93-98.

McIntosh AL, Gallegos AM, Atshaves BP, Storey SM, Kannoju D, Schroeder F. 2003. Fluorescence and multiphoton imaging resolve unique structural forms of sterol in membranes of living cells. J Biol Chem 278:6384-6403.

Mondal M, Mesmin B, Mukherjee S, Maxfield FR. 2009. Sterols are mainly in the cytoplasmic leaflet of the plasma membrane and the endocytic recycling compartment in CHO cells. Mol Biol Cell 20:581-588.

Mukherjee S, Zha X, Tabas I, Maxfield FR. 1998. Cholesterol distribution in living cells: Fluorescence imaging using dehydroergosterol as a fluorescent cholesterol analog. Biophys J 75:1915-1925.

Murray JM. 1998. Evaluating the performance of fluorescence microscopes. J Microsc 19:128-134.

Önfelt B, Nedvetzki S, Yanagi K, Davis DM. 2004. Cutting edge: Membrane nanotubes connect immune cells. J Immunol 173:15111513.

Petersen NO, Höddelius PL, Wiseman PW, Seger O, Magnusson KE. 1993. Quantitation of membrane receptor distributions by image correlation spectroscopy: Concept and application. Biophys J 65:1135-1146. 
Pipalia NH, Hao M, Mukherjee S, Maxfield FR. 2006. Sterol, protein and lipid trafficking in Chinese hamster ovary cells with NiemannPick type C1 defect. Traffic 8:130-141.

Rustom A. 2009. Hen or egg?: Some thoughts on tunneling nanotubes. Ann N Y Acad Sci 1178:129-136.

Rustom A, Saffrich R, Markovic I, Walther P, Gerdes H-H. 2004. Nanotubular highways for intercellular organelle transport. Science 303:1007-1010.

Salzmann NH, Maxfield FR. 1989. Fusion accessibility of endocytic compartments along the recycling and lysosomal endocytic pathways in intact cells. J Cell Biol 109:2097-2104.

Scheidt HA, Müller P, Herrmann A, Huster D. 2003. The potential of fluorescent and spin-labeled steroid analogs to mimic natural cholesterol. J Biol Chem 278:45563-45569.

Schrader M, Bahlmann K, Hell SW. 1997. Three-photon-excitation microscopy: Theory, experiment and applications. Optik 104:116124.

Slimane TA, Trugnan G, van IJzendoorn SC, Hoekstra D. 2003. Raftmediated trafficking of apical resident proteins occurs in both direct and transcytotic pathways in polarized hepatic cells: Role of distinct lipid microdomains. Mol Biol Cell 14:611-624.

Sormunen R, Eskelinen S, Lehto VP. 1993. Bile canaliculus formation in cultured HEPG2 cells. Lab Invest 68:652-662.

Stelzer EHK. 1998. Contrast, resolution, pixelation, dynamic range and signal-to-noise ratio: Fundamental limits to resolution in fluorescence light microscopy. J Microsc 189:15-24.

Tokumasu F, Fairhurst RM, Ostera GR, Brittain NJ, Hwang J, Wellems TE, Dvorak JA. 2005. Band 3 modifications in Plasmodium falciparum-infected AA and CC erythrocytes assayed by autocorrelation analysis using quantum dots. J Cell Sci 118:1091-1098.

van IJzendoorn SC, Hoekstra D. 1998. (Glyco)sphingolipids are sorted in sub-apical compartments in HepG2 cells: A role for non-Golgi related intracellular sites in the polarized distribution of (Glyco)sphingolipids. J Cell Biol 142:683-696.

van IJzendoorn SC, Hoekstra D. 1999. Polarized sphingolipid transport from the subapical compartment: Evidence for distinct sphingolipid domains. Mol Biol Cell 10:3449-3461.

Wiseman PW, Squier JA, Ellisman MH, Wilson KR. 2000. Two-photon image correlation spectroscopy and image cross-correlation spectroscopy. J Microsc 200:14-25.
Wojtal KA, de Vries E, Hoekstra D, van IJzendoorn SC. 2006. Efficient trafficking of MDR1/P-glycoprotein to apical canalicular plasma membranes in HepG2 cells requires PKA-RIIalpha anchoring and glucosylceramide. Mol Biol Cell 17:3638-3650.

Wüstner D. 2005. Improved visualization and quantitative analysis of fluorescent membrane sterol in polarized hepatic cells. J Microsc 220:47-64.

Wüstner D. 2007a. Fluorescent sterols as tools in membrane biophysics and cell biology. Chem Phys Lipids 146:1-25.

Wüstner D. 2007b. Plasma membrane sterol distribution resembles the surface topography of living cells. Mol Biol Cell 18:211-228.

Wüstner D. 2008. Free-cholesterol loading does not trigger phase separation of the fluorescent sterol dehydroergosterol in the plasma membrane of macrophages. Chem Phys Lipids 154:129-136.

Wüstner D, Færgeman NJ. 2008a. Chromatic aberration correction and deconvolution for UV sensitive imaging of fluorescent sterols in cytoplasmic lipid droplets. Cytometry A 73:727-744.

Wüstner D, Færgeman NJ. 2008b. Spatiotemporal analysis of endocytosis and membrane distribution of fluorescent sterols in living cells. Histochem Cell Biol 130:891-908.

Wüstner D, Mukherjee S, Maxfield FR, Müller P, Hermann A. 2001. Vesicular and nonvesicular transport of phosphatidylcholine in polarized HepG2 cells. Traffic 2:277-296.

Wüstner D, Herrmann A, Hao M, Maxfield FR. 2002. Rapid nonvesicular transport of sterol between the plasma membrane domains of polarized hepatic cells. J Biol Chem 277:30325-30336.

Wüstner D, Mondal M, Huang A, Maxfield FR. 2004. Different transport routes for high density lipoprotein and its associated free sterol in polarized hepatic cells. J Lipid Res 45:427-437.

Wüstner D, Mondal M, Tabas I, Maxfield FR. 2005. Direct observation of rapid internalization and intracellular transport of sterol by macrophage foam cells. Traffic 6:396-412.

Wüstner D, Landt Larsen A, Færgeman NJ, Brewer JR, Sage D. 2010. Selective visualization of fluorescent sterols in Caenorhabditis elegans by bleach-rate based image segmentation. Traffic 11:440-454.

Zhang W, McIntosh AL, Xu H, Wu D, Gruninger T, Atshaves B, Liu JC, Schroeder F. 2005. Structural analysis of sterol distributions in the plasma membrane of living cells. Biochemistry 44:2864-2884.

Zipfel WR, Williams RM, Webb WW. 2003. Nonlinear magic: Multiphoton microscopy in the biosciences. Nat Biotechnol 21:1369-1377. 